\title{
Adições à flora de briófitas de Mato Grosso do Sul, Brasil
}

\author{
Olga Yano ${ }^{1}$ e Cid José P. Bastos ${ }^{2,3}$
}

Recebido em 03/06/2003. Aceito em 19/11/2003

\begin{abstract}
RESUMO - (Adições à flora de briófitas de Mato Grosso do Sul, Brasil). Nesse levantamento foram encontrados 133 táxons no Estado de Mato Grosso do Sul, sendo um pertencente à divisão Anthocerotophyta em um gênero e uma família, 83 pertencentes à divisão Bryophyta, distribuídos em 54 gêneros e 27 famílias, e 49 à divisão Hepatophyta, distribuídos em 27 gêneros e 13 famílias. Destes, 100 (1 antócero, 59 musgos e 40 hepáticas) são novas citações para o Estado.
\end{abstract}

Palavras-chave: Anthocerotophyta, Bryophyta, Hepatophyta (Marchantiophyta), Mato Grosso do Sul

\begin{abstract}
Additions to the flora of bryophytes from Mato Grosso do Sul State, Brazil). In this taxonomic survey 133 taxa were found in Mato Grosso do Sul State, one belonging to Anthocerotophyta, distributed into one genus and one family, 83 belonging to Bryophyta, distributed into 54 genera and 27 families, and 49 belonging to Hepatophyta, distributed into 27 genera and 13 families. Of these, 100 (one hornworts, 59 mosses and 40 hepatics) are new records for the State.
\end{abstract}

Key words: Anthocerotophyta, Bryophyta, Hepatophyta (Marchantiophyta), Mato Grosso do Sul State

\section{Introdução}

O Estado de Mato Grosso do Sul localiza-se na região Centro-Oeste do Brasil. Possui área aproximada de $358.158,7 \mathrm{~km}^{2}$, recortados por vários rios e pela planície do Pantanal Matogrossense.

A vegetação da região é predominantemente cerrado (s.1.), com ocorrência de vegetações exuberantes das serras (Serra Amambaí, Serra da Bodoquena, Serra de Maracaju, Serra de São Jerônimo e uma pequena parte da Serra do Caiapó), e áreas de mata ciliar. Além disso, cada um dos ambientes pode formar os microambientes especiais para o crescimento das briófitas.

As briófitas no Mato Grosso do Sul são pouco estudadas, sendo relatadas em registros isolados, os quais podem ser encontrados em Yano (1981, 1984a, 1989 e 1995). Desses trabalhos foram relacionados 57 espécies de briófitas, sendo distribuídas em 46 gêneros e 31 famílias.

Até o momento não há trabalhos específicos de briófitas para a região, mas pode-se evidenciar o de Scremin-Dias et al. (1999) que relatam, dentre as plantas aquáticas, as briófitas como: Riccia fluitans L., Leptodictyon riparium (Hedw.) Warnst.,
Campylium hispidulum (Brid.) Mitt. e Hypnum amabile (Mitt.) Hampe, crescendo em diferentes rios da região.

O objetivo desse trabalho é o de contribuir para o melhor conhecimento da diversidade das briófitas e ampliar os dados sobre a distribuição geográfica das espécies no Estado de Mato Grosso do Sul e, conseqüentemente, no Brasil.

\section{Material e métodos}

Foram feitas algumas coletas em áreas de Mato Grosso do Sul, além de se analisar espécimes existentes no Herbário do Estado Maria Eneyda P. Kauffmann Fidalgo (SP) e material doado pelo Herbarium Riopretense da Universidade Estadual Júlio de Mesquita Filho, Campus de São José do Rio Preto (SJRP).

Na identificação das espécies foram utilizadas as seguintes referências: Bartram (1949), Bischler et al. (1963), Bischler (1969), Buck (1983), Florschütz (1964), Frahm (1991), Fulford (1976), Görts-Van Rijn (1996), Gradstein (1994), Hässel de Menéndez (1962), Hell (1969), Ireland \& Buck (1994), Lemos-Michel \& Yano (1998), Lindenberg (1844), Manuel (1977), Ochi (1980),

\footnotetext{
1 Instituto de Botânica, C. Postal 4005, CEP 01061-970, São Paulo, SP, Brasil

2 Universidade Federal da Bahia, Instituto de Biociências, Departamento de Botânica, Campus de Ondina, CEP 40170-280,Salvador, BA, Brasil

3 Autor para correspondência: cjpbasto@ufba.br
} 
Reese (1993), Reiner-Drehwald (2000), Schuster (1980), Sharp et al. (1994), Spence (1996), Stotler \& Crandall-Stotler (1974), Vanden Berghen (1976), Yano (1979, 1984b, 1992) e Zander (1993). Foi feita comparação com amostras identificadas por especialistas.

A classificação adotada baseou-se em Vitt (1984) para as Bryophyta, em Schuster (1984) para as Hepatophyta, e em Hasegawa (1994) para as Anthocerotophyta.

As famílias, os gêneros e as espécies estão listadas em ordem alfabética, dentro das divisões.

Os coletores do material briofítico O. Yano et al. são Iria H. Ishi, Geraldo A. Damaceno e M.P. Marcelli; os de M.R. Pietrobom-Silva et al. são F.R. Nonato e F. Firetti.

\section{Resultados}

Nesse levantamento, foram identificadas 133 espécies de briófitas, das quais apenas 1 antócero, 49 são hepáticas distribuídas em 13 famílias e 27 gêneros; sendo 83 musgos em 27 famílias e 54 gêneros. Destas, 100 espécies (1 antócero, 59 musgos e 40 hepáticas) são ocorrências novas para o Estado de Mato Grosso do Sul e estão sendo indicadas por asterisco $(*)$.

\section{ANTHOCEROTOPHYTA}

\section{ANTHOCEROTACEAE}

*Phaeoceros laevis (L.) Prosk.

Material examinado: BRASIL. Mato Grosso do Sul: munic. de Corumbá, Morro Tromba dos Macacos, paredão escorrendo água da cachoeira, $\pm 250 \mathrm{~m}$ alt., 2/XI/1993, O. Yano et al. 21134p.p. (SP 274128).

Cresce associada a Fissidens intramarginatus (Hampe) Mitt.

\section{HEPATOPHYTA}

\section{ANEURACEAE}

*Aneura pinguis L.

Material examinado: BRASIL. Mato Grosso do Sul: munic. de Corumbá, Morro Tromba dos Macacos, sobre pedra no riacho, $\pm 230 \mathrm{~m}$ alt., $2 / \mathrm{XI} / 1993, O$. Yano et al. 21102, 21109 (SP 274097; SP 274104).

*Riccardia cataractarum (Spruce) Hell

Material examinado: BRASIL. Mato Grosso do Sul: munic. de Corumbá, Morro Tromba dos Macacos, paredão escorrendo água da cachoeira, $\pm 250 \mathrm{~m}$ alt., 2/XI/1993, O. Yano et al. 21124, 21126p.p., 21127, 21132 (SP 274118; SP 274120; SP 274121; SP 274126).

Cresce associada a Telaranea nematodes (Aust.) Howe.

*Riccardia digitiloba (Spruce ex Steph.) Pagán, The Bryologist 42: 6.1939.

Material examinado: BRASIL. Mato Grosso do Sul: munic. de Selviria, Faz. do Cacildo, ca. 40km de Ilha Solteira em direção à Três Lagoas, margem de lagoa perto do cerradão, 24/X/1988, O. Yano \& $N$. Taroda 9288 (SP 191909); munic. de Cassilândia, ca. $51^{\circ} 37^{\prime} \mathrm{W}, 19^{\circ} 07^{\prime} \mathrm{S}$, ca. $400 \mathrm{~m}$ alt., MS-306 Cassilândia-Chapadão do Sul, ca. $5 \mathrm{~km}$ de Cassilândia, capões de cerrado, barranco úmido, 18/II/1996, M.R. Pietrobom-Silva et al. 2905 (SP 322788).

\section{CHONECOLEACEAE}

*Chonecolea doellingeri (Nees) Grolle

Material examinado: BRASIL. Mato Grosso do Sul: munic. de Rio Brilhante, sobre pau podre, 27/XI/1979, D.M. Vital 8566 (SP 133302); Rio Brilhante (21 $\left.30^{\circ} \mathrm{S}, 54^{\circ} 15^{\prime} \mathrm{W}\right)$, sobre tronco de Qualea sp., 27/XI/1979, D.M. Vital 8561p.p. (SP 147865); munic. de Três Lagoas, reserva Agroflorestal, sobre tronco de Qualea, $\pm 230 \mathrm{~m}$ alt., 12/X/1992, O. Yano \& M.P. Marcelli 17076 (SP 242220); munic. de Selviria, Faz. do Cacildo, $\pm 40 \mathrm{~km}$ de Ilha Solteira em direção à Três Lagoas, sobre cortiça de tronco no cerradão, 23/X/1984, O. Yano \& N. Taroda 9276 (SP 191897); idem, Estação Experimental da UNESP, sobre tronco podre no cerradão, 7/XI/1985, O. Yano \& M.R. Pereira-Noronha 9715 (SP 206420); idem, Faz. Agropecuária Santa Maria, base do tronco no cerradão, 7/XI/1985, O. Yano \& M.R. Pereira-Noronha 9736 (SP 206440); Campo Grande no campus da Univ. Federal de MS, sobre tronco de árvore, 8/IV/1998, $O$. Yano \& J. Xavier 25220, 25228, 25237 (SP 322037; SP 322045; SP 322052).

Cresce associada a Acrolejeunea torulosa (Lehm. \& Lindenb.) Schiffn.

\section{FOSSOMBRONIACEAE}

*Fossombronia brasiliensis Steph.

Material examinado: BRASIL. Mato Grosso do Sul: Ribas do Rio Pardo, barranco perto do rio, 25/I/1979, O. Yano 1325 (SP 133220). 
*Fossombronia paranapanemae Schiffn.

Material examinado: BRASIL. Mato Grosso do Sul: munic. de Rochedo (19 $\left.57^{\prime} \mathrm{S}, 5^{\circ} 07^{\prime} \mathrm{W}\right)$, bordos de um riacho, à sombra, 25/I/1979, D.M. Vital 8430, J. Váña (SP 133236).

\section{FRULLANIACEAE}

*Frullania ericoides (Nees) Mont.

Material examinado: BRASIL. Mato Grosso do Sul: Três Lagoas, praça perto da rua principal da usina, sobre tronco de Terminalia sp., 25/VIII/1983, O. Yano \& R.C. Compagnoli 8296 (SP 189732); idem, Novo Mundo, perto da cachoeira, sobre tronco de árvore, 17/III/1982, O. Yano 4035 (SP 174216); munic. de Jardim, ca. $52 \mathrm{~km}$ da cidade, sobre pau podre, 28/XI/1979, D.M. Vital 8583 (SP 133307); munic. de Corumbá, sobre tronco de Mangifera indica, 29/XI/1979, D.M. Vital 8597 (SP 133313); munic. de Miranda, sobre tronco de Mangifera indica, 3/XII/1979, D.M.Vital 8605 (SP 133316); Corumbá, ca. $77 \mathrm{~km}$ E-SE de Corumbá, sobre tronco de Sterculiaceae, 2/XII/1979, D.M. Vital 8617 (SP 133318); munic. de Ribas do Rio Pardo, sobre tronco de Terminalia catapa, na rua J.C. Garcia, 25/I/1979, O. Yano 1335p.p. (SP 133224); munic. de Selviria, Faz. Estação Experimental da UNESP, $\pm 18 \mathrm{~km}$ de Ilha Solteira, sobre tronco de árvore no cerradão, 22/X/1984, O. Yano \& J. Semir 9258 (SP 191879); munic. de Corumbá, Morro Tromba dos Macacos, sobre tronco de Mangifera, $\pm 210 \mathrm{~m}$ alt., 2/XI/1993, O. Yano et al. 21031 (SP 274029); idem, base de estipe de Bocaiúva, $\pm 210 \mathrm{~m}$ alt., 2/XI/1993, O. Yano et al. 21091 (SP 274086); Novo Mundo, perto da cachoeira, sobre tronco de Leguminosa, 18/III/1982, O. Yano 4033p.p. (SP 174215); munic. de Três Lagoas, reserva Agroflorestal, sobre tronco de árvore, $\pm 230 \mathrm{~m}$ alt., 12/IX/1992, O. Yano \& M.P. Marcelli 17018, 17026p.p., 17035, 17038, 17041, 17055, 17099 (SP 242163; SP 242171; SP 242180; SP 242183; SP 242186; SP 242200; SP 242241); idem, sobre galhos finos, 12/IX/1992, O. Yano \& M.P. Marcelli 17030 (SP 242175); idem, sobre tronco de Luehea, 12/IX/1992, O. Yano \& M.P. Marcelli 17071 (SP 242215); munic. de Selviria, Faz. do Cacildo, $\pm 40 \mathrm{~km}$ de Ilha Solteira em direção a Três Lagoa, sobre tronco de arbusto no cerradão, 23/X/1984, O. Yano \& N. Taroda 9274 (SP 191895); idem, galhos de arbusto no cerradão, 23/X/1984, O. Yano \& N. Taroda 9282 (SP 191903); idem, sobre tronco de Cecropia sp. na região pantanosa perto do cerradão, 24/X/1984, O. Yano \& N. Taroda 9289, 9294 (SP 191910; SP 191915); idem, na base do tronco na região pantanosa perto do cerradão, 24/X/1984, O. Yano \& N. Taroda 9295 (SP 191916); idem, sobre tronco de Byrsonima sp. no cerradão, 25/X/1984, O. Yano \& N. Taroda 9302, 9303 (SP 191922; SP 191923); idem, mata ciliar do Córrego da Véstia, sobre casca de tronco podre, 5/XI/1985, O. Yano \& M.R.P. Noronha 9573 (SP 206278); idem, sobre tronco, 5/XI/1985, O. Yano \& M.R.P. Noronha 9585, 9588, 9594, 9600, 9607, 9614, 9618 (SP 206290; SP 206293; SP 206299; SP 206305; SP 206312; SP 206319; SP 206323); idem, na base do tronco, 5/XI/1985, O. Yano \& M.R.P. Noronha 9613 (SP 206318); idem, Estação Experimental da UNESP, sobre tronco no cerradão, 7/XI/1985, O. Yano \& M.R. Pereira-Noronha 9725 (SP 206430); Campo Grande, reserva Biológica, Univ. Federal de MS, sobre tronco de arbusto, 8/XI/1985, $O$. Yano \& J. Xavier 25267, 25282, 25286 (SP 322080; SP 322095; SP 322099); idem, base do tronco de arbusto, 8/XI/1985, O. Yano \& J. Xavier 25277 (SP 322090).

Cresce associada a Aphanolejeunea truncatifolia Horik., Erpodium glaziovi Hampe, Mastigolejeunea auriculata (Wils.) Schiffn. e Papillaria nigrescens (Hedw.) A. Jaeger.

\section{Frullania gibbosa Nees}

Material examinado: BRASIL. Mato Grosso do Sul: munic. de Rio Brilhante ( $\left.21^{\circ} 30^{\prime} \mathrm{S}, 5^{\circ} 15^{\prime} \mathrm{W}\right)$, sobre tronco de Sapindaceae, no cerrado, 27/XI/1979, D.M. Vital 8564 (SP 133301); idem, ca. 40km de Guia Lopes de Laguna, sobre tronco de Sapotaceae, no cerradão, 27/XI/1979, D.M. Vital 8573 (SP 133303); munic. de Jardim, ca. $52 \mathrm{~km} \mathrm{~W}$ da cidade, sobre galhos de uma árvore, 28/XI/1979, D.M. Vital 8578 (SP 133304); munic. de Bonito, ca. $4 \mathrm{~km} \mathrm{~S}$ da cidade, sobre tronco de árvore isolada no cerrado, 28/XI/1979, D.M. Vital 8586p.p. (SP 133308); idem ca. 9km N. de Bonito, sobre tronco de Magonia sp. no cerrado, 28/XI/1979, D.M. Vital 8590 (SP 133310); munic. de Ribas do Rio Pardo, sobre tronco de árvore numa fazenda perto da reflorestadora Estância S.A., 25/I/1979, O. Yano 1340 (SP 133225); munic. de Rochedo, sobre um tronco curvado sobre um riacho Ribeirão Betina, 25/I/1979, D.M. Vital 8421 (SP 133232); idem, base of a tree

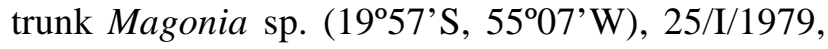
D.M. Vital 8424 (SP 133235); munic. de Três Lagoas, reserva Agroflorestal, sobre tronco de árvore, $\pm 230 \mathrm{~m}$ alt., 12/IX/1992, O. Yano \& M.P. Marcelli 17048 (SP 242193); munic. de Selviria, Faz. Estação 
Experimental da UNESP, $\pm 18 \mathrm{~km}$ da Ilha Solteira em direção a Ap. do Tabuado, sobre tronco no cerradão, 22/X/1984, O. Yano \& J. Semir 9253, 9259 (SP 191874; SP 191880); idem, Faz. do Cacildo, $\pm 40 \mathrm{~km}$ de Ilha Solteira em direção a Três Lagoas, sobre tronco no cerradão, 23/X/1984, O. Yano \& N. Taroda 9263 , 9264, 9268, 9270, 9275, 9280 (SP 191884; SP 191885; SP 191889; SP 191891; SP 191896; SP 191901); idem, base de pequenos arbustos no cerradão, 23/X/1984, O. Yano \& N. Taroda 9269 (SP 191890); idem, sobre tronco na região pantanosa perto do cerradão, 24/X/1984, O. Yano \& N. Taroda 9290 (SP 191911); idem, Estação Experimental da UNESP, sobre tronco vivo no cerradão, 7-XI-1985, O. Yano \& M.R. Pereira-Noronha 9726, 9723 (SP 206431; SP 206437); Campo Grande, reserva Biológica, Univ. Federal de MS, sobre fungo na mata úmida, 8/IV/1998, O. Yano \& J. Xavier 25248 (SP 322061).

Cresce associada a Acrolejeunea torulosa (Lehm. \& Lindenb.) Schiffn., Erythrodontium squarrosum (Müll. Hal.) Par. e Mastigolejeunea auriculata (Wils. \& Hook.) Schifffn.

*Frullania inflata Gott.

Material examinado: BRASIL. Mato Grosso do Sul: munic. de Corumbá, no morro de Urucum $\left(19^{\circ} 12^{\prime} S\right.$, $57^{\circ} 38^{\prime} \mathrm{W}$ ), sobre tronco de árvore junto a estrada da mina de ferro, 29/I/1979, D.M. Vital 8458 (SP 133240); munic. de Rochedo, sobre tronco de árvore, ao longo do rio Aquidauana ( $\left.20^{\circ} 08^{\prime} \mathrm{S}, 55^{\circ} 04^{\prime} \mathrm{W}\right), 25 / \mathrm{I} / 1979$, D.M. Vital 8419p.p. (SP 133230); munic. de Corumbá, Morro Tromba dos Macacos, sobre estipe de Bocaiúva, $\pm 210 \mathrm{~m}$ alt., 2/XI/1993, O. Yano et al. 21043 (SP 274040); idem, sobre tronco de arbusto, 210m alt., 2/XI/1993, O. Yano et al. 21089 (SP 274084).

Cresce associada a Dimerodontium mendozense Mitt.

*Frullania riojaneirensis (Raddi) Aongstr.

Material examinado: BRASIL. Mato Grosso do Sul: munic. de Bonito, ca. $22 \mathrm{~km}$ da cidade, sobre tronco fino na encosta de morro, 28/XI/1979, D.M. Vital 8595 (SP 133311); munic. de Selviria, Faz. Estação Experimental da UNESP, $\pm 18 \mathrm{~km}$ de Ilha Solteira, sobre tronco no cerradão, 22/X/1984, O. Yano \& $J$. Semir 9254 (SP 191875); munic. de Corumbá, Morro Tromba dos Macacos, base do estipe de Bocaiúva, $\pm 210 \mathrm{~m}$ alt., 2/XI/1993, O. Yano et al. 21073, 21077 (SP 274069; SP 274073); munic. de Três Lagoas, reserva Agroflorestal, sobre tronco de árvore na mata, $\pm 230 \mathrm{~m}$ alt., 12/IX/1992, O. Yano \& M.P. Marcelli 17053, 17054, 17069, 17075 (SP 242198; SP 242199; SP 242214; SP 242219); idem, sobre tronco de Luehea, 12/IX/1992, O. Yano \& M.P. Marcelli 17062p.p. (SP 242207); munic. de Selviria, Faz. Estação Experimental da UNESP, $\pm 18 \mathrm{~km}$ de Ilha Solteira em direção a Ap. do Tabuado, sobre galhos de árvore no cerradão, 22/X/1984, O. Yano \& J. Semir 9249 (SP 191870); idem, sobre tronco no cerradão, 22/X/1984, O. Yano \& J. Semir 9717, 9729 (SP 206422; SP 206434); Campo Grande, campus da Univ. Federal de MS, sobre tronco de árvore, 8/IV/1988, O. Yano \& J. Xavier 25231, 25256 (SP 322047; SP 322069).

Cresce associada a Frullania dilatata (L.) Dumort. e Lejeunea trinitensis Lindenb.

*Frullania supradecomposita (Lehm. \& Lindenb.) Lehm. \& Lindenb.

Material examinado: BRASIL. Mato Grosso do Sul: munic. de Corumbá, Morro Tromba dos Macacos, sobre estipe de Bocaiúva, $\pm 210 \mathrm{~m}$ alt., 2/XI/1993, $O$. Yano et al. 21045 (SP274042).

\section{GEOCALYCACEAE}

*Chiloscyphus sp.

Material examinado: BRASIL. Mato Grosso do Sul: Ribas do Rio Pardo, $\pm 71 \mathrm{~km}$ depois de Campo Grande, barranco do rio, 25/I/1979, O. Yano 1328 (SP 133221).

\section{*Lophocolea bidentata (L.) Dumort.}

Material examinado: BRASIL. Mato Grosso do Sul: munic. de Ribas do Rio Pardo, 16km da cidade, margem do rio, 25/I/1979, O. Yano 1343p.p., 1347p.p. (SP 133226; SP 133227); munic. de Alto Taquari, ca. $53^{\circ} 17^{\prime} \mathrm{N}, 17^{\circ} 50^{\prime}$ 'S, ca. $600-650 \mathrm{~m}$ alt., ca. $25 \mathrm{~km}$ a sudeste da cidade, Córrego da Laje, paredão rochoso úmido, 21/II/1996, M.R. Pietrobom-Silva et al. 3013p.p. (SP 322838).

Cresce associada a Lopholejeunea nigricans (Lindenb.) Schiffn., Neckeropsis undulata (Hedw.) Reichardt, Racopilum tomentosum (Hedw.) Brid. e Zelometeorium patulum (Hedw.) Manuel.

\section{JUNGERMANNIACEAE}

Jungermannia hyalina Lyell in Hook.

Material examinado: BRASIL. Mato Grosso do

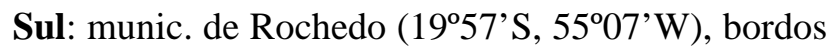


de um riacho, 25/I/1979, D.M. Vital 8431, J. Váña (SP 133237).

\section{LEJEUNEACEAE}

Acrolejeunea emergens (Mitt.) Steph.

Material examinado: BRASIL. Mato Grosso do Sul: munic. de Rochedo, sobre tronco curvado sobre um riacho, 25/I/1979, D.M. Vital 8423 (SP 133234).

*Acrolejeunea torulosa (Lehm. \& Lindenb.) Schiffn.

Material examinado: BRASIL. Mato Grosso do Sul: munic. de Ribas do Rio Pardo, $\pm 71 \mathrm{~km}$ de Campo Grande em direção à Ribas do Rio Pardo, sobre tronco de árvore, mata de galeria, 25/I/1979, O. Yano 1331 (SP 133223); munic. de Rochedo, sobre tronco curvado sobre Ribeirão Betina, 25/I/1979, D.M. Vital 8421p.p. (SP 133232); munic. de Selviria, Faz. Estação Experimental da UNESP, $\pm 18 \mathrm{k}$, de Ilha Solteira, sobre tronco, no cerradão, 22/X/1984, O. Yano \& J. Semir 9255, 9247, 9250, 9252 (SP 191876; SP 191868; SP 191871; SP 191873); idem, Faz. do Cacildo, $\pm 40 \mathrm{~km}$ de Ilha Solteira em direção a Três Lagoas, sobre tronco no cerradão, 23/X/1984, O. Yano \& N. Taroda 9266 (SP 191887); idem, sobre cortiça de tronco no cerradão, 23/X/1984, O. Yano \& $N$. Taroda 9271 (SP 191892); idem, sobre tronco no cerradão, 25/X/1984, O. Yano \& N. Taroda 9301 (SP 191921); idem, Córrego da Véstia, sobre tronco, mata ciliar, 5/XI/1985, O. Yano \& M.R.P. Noronha 9586 (SP 206291); idem, Faz. Agropecuária Santa Maria, sobre tronco no cerradão, 7/XI/1985, O. Yano \& M.R. Pereira-Noronha 9734, 9742, 9750 (SP 206438; SP 206446; SP 206454); idem, base do tronco no cerradão, 7/XI/1985, O. Yano \& M.R. Pereira Noronha 9747 (SP 206451).

Cresce associada a Chonecolea doellingeri (Nees) Grolle, Frullania gibbosa Nees e Mastigolejeunea auriculata (Wils. \& Hook.) Schiffn.

\section{*Aphanolejeunea microscopica (Tayl.) A. Evans}

Material examinado: BRASIL. Mato Grosso do Sul: Campo Grande, sobre tronco de Inga sp., praça de República, 27/I/1979, D.M. Vital 8446p.p. (SP 133239); munic. de Rochedo, sobre tronco de laranjeira, ao longo do rio Aquidauana (2008'S, 5504'W), 25/I/1979, D.M. Vital 8420p.p. (SP 133231).

Cresce associada a Cololejeunea minutissima (Sm.) Schiffn. e Erpodium glazioui Hampe
*Aphanolejeunea truncatifolia Horik.

Material examinado: BRASIL. Mato Grosso do Sul: munic. de Costa Rica, ca. 53 ${ }^{\circ} 13^{\prime} \mathrm{W}, 18^{\circ} 47^{\prime} \mathrm{S}$, ca. 450m alt., Ribeirão Sucuruí, aos fundos da cidade Costa Rica, epífila de Bolbitis serratifolia, interior da mata de encosta, 20/II/1996, M.R. Pietrobom-Silva et al. 2979 (SP 322813); munic. de Três Lagoas, reserva Agroflorestal, sobre liana na mata, $\pm 230 \mathrm{~m}$ alt., 12/IX/1992, O. Yano \& M.P. Marcelli 17029, 17050 (SP 242167; SP 242195); idem, base do tronco de árvore, 12/IX/1992, O. Yano \& M.P. Marcelli 17043 (SP 242188); idem, epífila de arbusto na mata, 12/IX/1992, O. Yano \& M.P. Marcelli 17096 (SP 242238); Campo Grande, reserva Biológica, Unvi. Federal de MS, sobre tronco de árvore da reserva, 8/IV/1998, O. Yano \& J. Xavier 25239 (SP 322054); idem, sobre tronco de Lauraceae, mata úmida, 8/IV/1998, O. Yano \& J. Xavier 25273 (SP 322086); idem, sobre tronco podre, 8/IV/1998, O. Yano \& J. Xavier 25294 (SP 322107).

Cresce associada a Cololejeunea cardiocarpa (Mont.) A. Evans, Frullania ericoides (Nees) Mont. e Lejeunea trinitensis Lindenb.

*Archilejeunea parviflora (Nees) Schiffn.

Material examinado: BRASIL. Mato Grosso do Sul: munic. de Corumbá, Morro Tromba dos Macacos, base do tronco de arbusto na mata, $\pm 230 \mathrm{~m}$ alt., 2/XI/1993, O. Yano et al. 21111 (SP 274106); idem, sobre pedra exposta, $\pm 230 \mathrm{~m}$ alt., $2 / \mathrm{XI} / 1993$, O. Yano et al. 21115 (SP 274110).

*Brachiolejeunea laxifolia (Tayl.) Schiffn.

Material examinado: BRASIL. Mato Grosso do Sul: munic. de Selviria, Faz. Estação Experimental da UNESP, $\pm 18 \mathrm{~km}$ de Ilha Solteira, sobre cortiça do tronco no cerradão, 22/X/1984, O. Yano \& J. Semir 9260 (SP 191881).

\section{*Bryopteris filicina (Sw.) Nees}

Material examinado: BRASIL. Mato Grosso do Sul: munic. de Costa Rica, ca. 53 ${ }^{\circ} 14^{\prime} \mathrm{W}, 1^{\circ} 47^{\prime} \mathrm{S}$, ca. $715 \mathrm{~m}$ alt., rod. MS Cassilândia-Alto Araguaia, povoado Laje, Rio Laje, sobre tronco, 5/IX/1993, M.R. PietrobomSilva \& C.A. Nobile 1074 (SP 322847); munic. de Alto Taquari, ca. $53^{\circ} 17^{\prime} \mathrm{W}, 17^{\circ} 50^{\prime} \mathrm{S}$, ca. $600-650 \mathrm{~m}$ alt., ca. $25 \mathrm{~km}$ a sudeste da cidade, paredão úmido, 21/II/1996, M.R. Pietrobom-Silva et al. 3011p.p. (SP 322837).

Cresce associada a Lejeunea autoica R.M. Schust., Porotrichum longirostre (Hook.) Mitt. 
*Bryopteris fruticulosa T. Taylor

Material examinado: BRASIL. Mato Grosso do Sul: munic. de Costa Rica, ca. 53 ${ }^{\circ} 13^{\prime} \mathrm{W}, 18^{\circ} 47^{\prime} \mathrm{S}$, povoado Laje, ca. 550m alt., MS-306 Cantina Costa Rica-Capela, cachoeira do Rio Laje, barranco rochoso, 20/II/1996, M.R. Pietrobom-Silva et al. 2978 a (SP 322812); munic. de Rio Verde de Mato Grosso, ca. $54^{\circ} 53^{\prime}$ W, $18^{\circ} 55^{\prime}$ 'S, ca. $400 \mathrm{~m}$ alt., rod. Sete QuedasRio Negro, ca. 30km da cidade, Fazenda Mirante, Cachoeira do Cervo, Serra Pimenteira, barranco úmido, 31/X/1993, C.E. Rodrigues Jr. \& M.R. PietrobomSilva 735 (SP 322855); munic. de Alto Taquari, ca. $53^{\circ} 17^{\prime} \mathrm{W}, 17^{\circ} 50^{\prime} \mathrm{S}$, ca. $600-650 \mathrm{~m}$ alt., ca. $25 \mathrm{~km}$ a sudeste da cidade, sobre tronco de arvoreta, 21/II/1996, M.R. Pietrobom-Silva et al. 3020 (SP 322844).

Cheilolejeunea rigidula (Mont.) R.M. Schust.

Material examinado: BRASIL. Mato Grosso do Sul: munic. de Costa Rica, ca. 53 ${ }^{\circ} 13^{\prime} \mathrm{W}, 18^{\circ} 47^{\prime}$ 'S, ca. $450 \mathrm{~m}$ alt., Ribeirão Sucuruí, aos fundos da cidade de Costa Rica, sobre tronco em decomposição, 20/II/1996, M.R. Pietrobom-Silva et al. 2983p.p. (SP 322817); munic. de Três Lagoas, reserva Agroflorestal, sobre tronco de árvore, $\pm 230 \mathrm{~m}$ alt., $12 / \mathrm{IX} / 1992, O$. Yano \& M.P. Marcelli 17031, 17042, 17092 (SP 242176; SP 242187; SP 242234); idem, base do tronco de árvore, 12/IX/1992, O. Yano \& M.P. Marcelli 17081 (SP 242225); munic. de Selviria, Faz. do Cacildo, $\pm 40 \mathrm{~km}$ de Ilha Solteira em direção a Três Lagoas, sobre tronco de árvore na região pantanosa perto do cerradão, 24/X/1984, O. Yano \& N. Taroda 9286 (SP 191907); idem, Córrego da Véstia, sobre tronco, 5/XI/1985, O. Yano \& M.R.P. Noronha 9582, 9591, 9597(SP 206287; SP 206296; SP 206301); idem, sobre tronco de Myrtaceae, 5/XI/1985, O. Yano \& M.R.P. Noronha 9587 (SP 206292).

Cresce associada a Calymperes afzelii Sw.

*Cheilolejeunea trifaria (Reinw. et al.) Mizut.

Material examinado: BRASIL. Mato Grosso do Sul: munic. de Aparecida do Taboado, ca. $51^{\circ} 05^{\prime} \mathrm{W}$, $20^{\circ} 06^{\prime}$ S, ca. $300 \mathrm{~m}$ alt., rod. BR-158 Aparecida do Taboado-Paranaíba, ca. 20km de Ap. do Taboado, capões de cerradão, sobre tronco de peroba, 17/II/1996, M.R. Pietrobom-Silva et al. 2832 (SP 322776); munic. de Cassilândia, ca. $51^{\circ} 37^{\prime} \mathrm{W}, 1^{\circ} 07^{\prime}$ 'S, ca. $400 \mathrm{~m}$ alt., MS-306 Cassilândia-Chapadão do Sul, ca. 5km de Cassilândia, capões de cerrado, sobre tronco de árvore, 18/II/1996, M.R. Pietrobom-Silva et al. 2904 (SP 322787); munic. de Indaiá do Sul (= Chapéu Azul) ca. $52^{\circ} 23^{\prime} \mathrm{W}, 18^{\circ} 57^{\prime} \mathrm{S}$, ca. $500 \mathrm{~m}$ alt., Cachoeira dos fundos da cidade, capões de cerrado, sobre tronco, 18/II/1996, M.R. Pietrobom-Silva et al. 2934p.p. (SP 322790).

Cresce associada a Zelometeorium patulum (Hedw.) Manuel.

* Cololejeunea cardiocarpa (Mont.) A. Evans

Material examinado: BRASIL. Mato Grosso do Sul: munic. de Alto Taquari, ca. $53^{\circ} 17^{\prime} \mathrm{W}$ e $17^{\circ} 50^{\prime} \mathrm{S}$, ca. $600-650 \mathrm{~m}$ alt., ca. $25 \mathrm{~km}$ à sudeste da cidade, Córrego da Laje, epífila de Bolbitis serratifolia, 21/II/1996, M.R. Pietrobom-Silva et al. 3017 (SP 322841); munic. de Três Lagoas, reserva Agroflorestal, sobre folha de arbusto, $\pm 230 \mathrm{~m}$ alt., 12/IX/1992, O. Yano \& M.P. Marcelli 17037, 17059, 17078 (SP 242182; SP 242204; SP 242222); idem, sobre tronco de árvore, 12/IX/1992, O. Yano \& M.P. Marcelli 17059p.p. (SP 242204); idem, base do tronco de árvore, 12/IX/1992, O. Yano \& M.P. Marcelli 17065 (SP 242210); idem, epífila de arbusto na mata, $\pm 230 \mathrm{~m}$ alt., 12/IX/1992, O. Yano \& M.P. Marcelli 17093, 17095, 17101, 17103, 17109, 17111, 17119, 17122, 17124 (SP 242235; SP 242237; SP 242243; SP 242245; SP 242249; SP 242251; SP 242258; SP 242261; SP 242263); Campo Grande, reserva Biológica, Univ. Federal de MS, base do tronco podre, mata úmida, 8/IV/1998, O. Yano \& J. Xavier 25266 (SP 322079); idem, epífila de arbusto, 8/IV/1998, $O$. Yano 25289, 25290, 25296, 25297, 25298, 25299, 25300, 25301, 25302 (SP 322102; SP 322103; SP 322109; SP 322110; SP 322111; SP 322112; SP 322113; SP 322114; SP 322115). Horik.

Cresce associada a Aphanolejeunea truncatifolia

\section{*Cololejeunea minutissima (Sm.) Schiffn.}

Material examinado: BRASIL. Mato Grosso do Sul: munic. de Rochedo, sobre tronco de laranjeira, ao longo do rio Aquidauana ( $\left.20^{\circ} 08^{\prime} \mathrm{S}, 55^{\circ} 04^{\prime} \mathrm{W}\right), 25 / \mathrm{I} / 1979$, D.M. Vital 8420p.p. (SP 133231); munic. de Três Lagoas, reserva Agroflorestal, sobre tronco queimado, $\pm 230 \mathrm{~m}$ alt., 12/IX/1992, O. Yano \& M.P. Marcelli 12051 (SP 242196); idem, sobre tronco de árvore, 12/IX/1992, O. Yano \& M.P. Marcelli 17052 (SP 242197); munic. de Selviria, Faz. do Cacildo, $\pm 40 \mathrm{~km}$ de Ilha Solteira em direção a Três Lagoas, sobre tronco no cerradão, 23/X/1984, O. Yano \& N. Taroda 9278 (SP 191899).

Cresce associada a Aphanolejeunea microscopica (Tayl.) A. Evans. 
*Lejeunea autoica R.M. Schust.

Material examinado: BRASIL. Mato Grosso do Sul: munic. de Alto Taquari, ca. $53^{\circ} 17^{\prime} \mathrm{W}-17^{\circ} 50^{\prime} \mathrm{S}$, ca. $600-650 \mathrm{~m}$ alt., ca. $25 \mathrm{~km}$ a sudeste da cidade, paredão rochoso úmido, 21/II/1996, M.R. PietrobomSilva et al. 3011p.p. (SP 322837).

Cresce associada a Bryopteris filicina (Sw.) Nees e Porotrichum longirostre (Hook.) Mitt.

*Lejeunea cardoti Steph.

Material examinado: BRASIL. Mato Grosso do Sul: munic. de Corumbá, Morro Tromba dos Macacos, sobre tronco de Leguminosae na mata, $\pm 210 \mathrm{~m}$ alt., 2/X/1993, O. Yano et al. 21087 (SP 274082); munic. de Três Lagoas, reserva Agroflorestal, base do tronco de árvore, $\pm 230 \mathrm{~m}$ alt., 12/IX/1992, O. Yano \& M.P. Marcelli 17017 (SP 242162); Campo Grande, reserva Biológica, Univ. Federal de MS, base do tronco de árvore, mata úmida, 8/IV/1998, O. Yano \& J. Xavier 25258 (SP 322071).

\section{*Lejeunea flava (Sw.) Nees}

Material examinado: BRASIL. Mato Grosso do Sul: munic. de Costa Rica ca. 53 $13^{\circ} \mathrm{W}, 18^{\circ} 47^{\prime} \mathrm{S}$, povoado Laje, ca. 550m alt., MS-306 Cantina Costa Rica-Capela, cachoeira do Rio Laje, sobre tronco de árvore, 20/II/1996, M.R. Pietrobom-Silva et al. 2978p.p. (SP 322811); munic. de Jardim, ca. $52 \mathrm{~km} \mathrm{~W}$ da cidade, sobre pau podre, 28/XI/1979, D.M. Vital 8580 (SP 133305); munic. de Selviria, Faz. do Cacildo, $\pm 40 \mathrm{~km}$ de Ilha Solteira em direção a Três Lagoas, base do tronco vivo no cerradão, 25/X/1984, O. Yano \& N. Taroda 9305 (SP 191925); Campo Grande, reserva Biológica, Univ. Federal de MS, sobre tronco de árvore na mata úmida, 8/IV/1998, O. Yano \& J. Xavier 25253 (SP 322066); idem, base do tronco de árvore, 8/IV/1998, O. Yano \& J. Xavier 25262 (SP 322075).

Cresce associada a Octoblepharum albidum Hedw. var. albidum.

\section{*Lejeunea glaucescens Gottsche}

Material examinado: BRASIL. Mato Grosso do Sul: munic. de Aparecida do Taboado, ca. 51 $05^{\circ}$ 'W$20^{\circ} 06$ 'S, ca. $300 \mathrm{~m}$ alt., rod. BR-158 Aparecida do Taboado-Paranaíba, ca. 20km de Ap. do Taboado, capões de cerradão, sobre tronco em decomposição, 17/II/1996, M.R. Pietrobom-Silva et al. 2830p.p. (SP 322774); munic. de Três Lagoas, reserva Agroflorestal, base do tronco na mata, $\pm 230 \mathrm{~m}$ alt.,
12/IX/1992, O. Yano \& M.P. Marcelli 17087 (SP 242230); idem, sobre tronco de árvore, 12/IX/1992, O. Yano \& M.P. Marcelli 17118, 1992 (SP 242257); Campo Grande, reserva Biológica, Univ. Federal de MS, base do tronco podre, 8/IV/1998, O. Yano \& J. Xavier 25284 (SP 322097).

Cresce associada a Chryso-hypnum diminutivum (Hampe) W.R. Buck.

\section{*Lejeunea laetevirens Nees \& Mont.}

Material examinado: BRASIL. Mato Grosso do Sul: Campo Grande, sobre tronco de Licania sp., na praça Afonso Pena, 22/I/1979, D.M. Vital 8409 (SP 133228); idem, sobre tronco de Inga sp., praça próximo ao QG do exército, 31/I/1979, D.M. Vital 8461p.p. (SP 133241); munic. de Jardim, ca. 52km da cidade, sobre pau podre, 28/XI/1979, D.M. Vital 8582 (SP 133306); munic. de Três Lagoas, reserva Agroflorestal, base do tronco de árvore, $\pm 230 \mathrm{~m}$ alt., 12/IX/1992, O. Yano \& M.P. Marcelli 17040 (SP 242185); munic. de Selviria, Faz. do Cacildo, $\pm 40 \mathrm{~km}$ de Ilha Solteira em direção a Três Lagoas, sobre tronco de Cecropia sp. na região pantanosa perto do cerradão, 24/X/1984, O. Yano \& N. Taroda 9287 (SP 191908); idem, sobre tronco na região pantanosa perto do cerradão, 24/X/1984, O. Yano \& N. Taroda 9292 (SP 191913).

Cresce associada a Cheilolejeunea rigidula (Nees \& Mont.) R.M. Schust., Fabronia ciliaris var. polycarpa (Hook.) W.R. Buck e Frullania ericoides (Nees) Mont.

\section{*Lejeunea minutiloba A. Evans}

Material examinado: BRASIL. Mato Grosso do Sul: munic. de Corumbá, Morro Tromba dos Macacos, sobre raízes perto do riacho, $\pm 210 \mathrm{~m}$ alt., 2/XI/1993, O. Yano et al. 21038 (SP 274036); idem, sobre pedra, 2/XI/1993, O. Yano et al. 21039 (SP274037); idem no paredão escorrendo água, 2/XI/1993, O. Yano et al. 21118 (SP 274112); munic. de Três Lagoas, reserva Agroflorestal, base do tronco $\pm 230 \mathrm{~m}$ alt., 12/IX/1992, O. Yano \& M.P. Marcelli 17049, 17064, 17079, 17091, 17094 (SP 242194; SP 242209; SP 242223; SP 242233; SP 242236); idem, sobre tronco de árvore na mata, 12/IX/1992, O. Yano \& M.P. Marcelli 17100, 17115 (SP 242242; SP 242254); idem, sobre tronco de Lauraceae na mata, 12/IX/1992, O. Yano \& M.P. Marcelli 17105 (SP 242247); idem, sobre tronco podre, 12/IX/1992, O. Yano \& M.P. Marcelli 17112, 17120 (SP 242252; SP 242259); idem, sobre tronco de Copaífera, 12/IX/1992, O. Yano \& M.P. Marcelli 
17113p.p. (SP 242253); Campo Grande, reserva Biológica, Univ. Federal de MS, sobre tronco podre caído na mata, 8/IV/1998, O. Yano \& J. Xavier 25244 (SP 322059).

\section{*Lejeunea phyllobola Nees \& Mont.}

Material examinado: BRASIL. Mato Grosso do Sul: munic. de Corumbá, Morro Tromba dos Macacos, base do estipe de Bocaiúva, $\pm 210 \mathrm{~m}$ alt., 2/XI/1993, O. Yano et al. 21072 (SP 274068); idem, sobre pedra exposta na mata, $\pm 230 \mathrm{~m}$ alt., 2/XI/1993, O. Yano et al. 21114 (SP 274109); munic. de Três Lagoas, reserva Agroflorestal, sobre tronco de árvore, $\pm 230 \mathrm{~m}$ alt., 12/IX/1992, O. Yano \& M.P. Marcelli 17016 (SP 242161); munic. de Selviria, Córrego da Véstia, sobre tronco, mata ciliar, 5/XI/1985, O. Yano \& M.R.P. Noronha 9577, 9578p.p., 9581, 9583, 9601, 9602 (SP 206282; SP 206283; SP 206286; SP 206288; SP 206306; SP 206307); Campo Grande, reserva Biológica, Univ. Federal de MS, sobre tronco de arbusto, mata úmida, 8/IV/1998, O. Yano \& J. Xavier 25276 (SP 322089); idem, base do tronco de arbusto, 8/IV/1998, O. Yano \& J. Xavier 25293 (SP 322106). Mitt.

Cresce associada a Isopterygium tenerum (Sw.)

\section{*Lejeunea setiloba Spruce}

Material examinado: BRASIL. Mato Grosso do Sul: Campo Grande, reserva Biológica, Univ. Federal de MS, sobre tronco de arbusto, mata úmida, 8/IV/1998, O. Yano \& J. Xavier 25270 (SP 322083); munic. de Corumbá, Morro Tromba dos Macacos, sobre tronco de Annonaceae na mata, $\pm 210 \mathrm{~m}$ alt., 2/XI/1993, O. Yano et al. 21080 (SP 274075).

\section{*Lejeunea trinitensis Lindenb.}

Material examinado: BRASIL. Mato Grosso do Sul: munic. de Rochedo, sobre tronco de árvore, ao longo do rio Aquidauana ( $\left.20^{\circ} 08^{\prime} \mathrm{S}, 55^{\circ} 04^{\prime} \mathrm{W}\right), 25 / \mathrm{I} / 1979$, D.M. Vital 8419p.p. (SP 133230); munic. de Corumbá, Morro Tromba dos Macacos, sobre tronco de árvore, $\pm 210 \mathrm{~m}$ alt., 2/XI/1993, O. Yano et al. 21040, 21057 (SP 274038; SP 274054); munic. de Três Lagoas, reserva Agroflorestal, sobre tronco de Luehea, $\pm 230 \mathrm{~m}$ alt., 12/IX/1992, O. Yano \& M.P. Marcelli 17062p.p. (SP 242207); Campo Grande, reserva Biológica, Univ. Federal de MS, base do tronco de arbusto, 8/IV/1998, O. Yano \& J. Xavier 25268, 25295 (SP 322081; SP 322108); idem, base do tronco de Piperaceae, mata úmida, 8/IV/1998, O. Yano \& J. Xavier 25278 (SP 322091); idem, sobre tronco podre, mata úmida,
8/IV/1998, O. Yano \& J. Xavier 25294p.p. (SP 322107).

Cresce associada a Aphanolejeunea truncatifolia Horik., Dimerodontium mendozense Mitt. e Frullania riojaneirensis (Raddi) Aongstr.

*Leptolejeunea exocellata (Spruce) A. Evans

Material examinado: BRASIL. Mato Grosso do Sul: munic. de Costa Rica, ca. 53 ${ }^{\circ} 13^{\prime} \mathrm{W}, 18^{\circ} 47^{\prime} \mathrm{S}$, ca. $40 \mathrm{~m}$ alt., Ribeirão Sucuruí, aos fundos da cidade de Costa Rica, epífila de Adiantum sp., 20/II/1996, M.R. Pietrobom-Silva et al. 2980 (SP 322814); munic. de Alto Taquari, ca. $53^{\circ} 17^{\prime} \mathrm{W}, 17^{\circ} 50^{\prime} \mathrm{S}$, ca. $600-650 \mathrm{~m}$ alt., ca. $25 \mathrm{~km}$ a sudeste da cidade, epífila de Adiantum sp., 21/II/1996, M.R. Pietrobom-Silva et al. 3022 (SP 322846).

*Lopholejeunea nigricans (Lindenb.) Schiffn.

Material examinado: BRASIL. Mato Grosso do Sul: Ribas do Rio Pardo, $16 \mathrm{~km}$ antes da cidade, margem do rio, 25/I/1979, O. Yano 1343p.p. (SP 133226).

Cresce assocoada a Lophocolea bidentata (L.) Dumort.

Mastigolejeunea auriculata (Wils. \& Hook.) Schiffn.

Material examinado: BRASIL. Mato Grosso do Sul: Novo Mundo, perto da cachoeira, sobre tronco de árvore, 18/III/1982, O. Yano 4036 (SP 174217); munic. de Bonito, sobre tronco de Magonia, no cerrado, 28/XI/1979, D.M. Vital 8589 (SP 133309); munic. de Ribas do Rio Pardo, $\pm 71 \mathrm{~km}$ de Campo Grande, sobre tronco de Myrtaceae próximo ao rio, 25/I/1979, O. Yano 1330 (SP 133222); munic. de Rochedo $\left(19^{\circ} 57^{\prime} \mathrm{S}, 55^{\circ} 07^{\prime} \mathrm{W}\right)$, bordos de um riacho à sombra, 25/I/1979, D.M. Vital 8432 (SP 133238); idem, sobre tronco curvado sobre ribeirão Betina, 25/I/1979, D.M. Vital 8422 (SP 133233); munic. de Corumbá, Morro Tromba dos Macacos, sobre tronco de Bocaiúva, $\pm 210 \mathrm{~m}$ alt., 2/XI/1993, O. Yano et al. 21055, 21082 (SP 274052; SP 274077); munic. de Três Lagoas, reserva Agroflorestal, sobre tronco de árvore, $\pm 230 \mathrm{~m}$ alt., 12/IX/1992, O. Yano \& M.P. Marcelli 17026p.p., 17080,17110 (SP 242171; SP 242224; SP 242250); idem, base do tronco de árvore, 12/IX/1992, O. Yano \& M.P. Marcelli 17033, 17044 (SP 242178; SP 242189); idem, sobre tronco de Lauraceae, 12/IX/1992, O. Yano \& M.P. Marcelli 17108 (SP 242248); munic. de Selviria, 5/XI/1985, O. Yano \& M.R.P. Noronha 9580, 9589, 9590, 9604, 9616 (SP 206285; SP 206294; SP 206295; SP 206309; SP 206321). 
Cresce associada a Acrolejeunea torulosa (Lehm. \& Lindenb.) Schiffn., Frullania ericoides (Nees) Mont. e Frullania gibbosa Nees.

Microlejeunea epiphylla Bischler

Material examinado: BRASIL. Mato Grosso do Sul: munic. de Corumbá, Morro Tromba dos Macacos, sobre estipe de Bocaiúva na mata, $\pm 210 \mathrm{~m}$ alt., 2/XI/1993, O. Yano et al. 21088 (SP274083).

*Schiffneriolejeunea polycarpa (Nees) Gradst.

Material examinado: BRASIL. Mato Grosso do Sul: munic. de Bonito, ca. 22km N-NW da cidade, sobre tronco na encosta do morro, 28/XI/1979, D.M. Vital 8596p.p. (SP 133312); munic. de Corumbá, Morro Tromba dos Macacos, sobre estipe de Bocaiúva na mata, $\pm 210 \mathrm{~m}$ alt., 2/XI/1993, O. Yano et al. 21083p.p.(SP 274078); munic. de Selviria, Faz. da Estação Experimental da UNESP, $\pm 18 \mathrm{~km}$ de Ilha Solteira em direção ao Ap. do Tabuado, sobre tronco no cerradão, 22/X/1984, O. Yano \& J. Semir 9244 (SP 191866); idem, Faz. do Cacildo, $\pm 40 \mathrm{~km}$ de Ilha Solteira em direção a Três Lagoas, sobre tronco na região pantanosa perto do cerradão, 24/X/1984, O. Yano \& N. Taroda 9293 (SP 191914).

Cresce associada a Entodontopsis leucostega (Brid.) W.R. Buck \& Ireland e Helicophyllum torquatum (Hook.) Brid.

\section{LEPIDOZIACEAE}

\section{*Telaranea nematodes (Aust.) Howe}

Material examinado: BRASIL. Mato Grosso do Sul: munic. de Selviria, Faz. do Cacildo, ca 40km de Ilha Solteira em direção a Três Lagoas, sobre raízes aéreas de pteridófitas na região pantanosa perto do cerradão, 24/X/1984, O. Yano \& N. Taroda 9283 (SP 191904); munic. de Corumbá, Morro Tromba dos Macacos, paredão escorrendo água da cachoeira, $\pm 250 \mathrm{~m}$ alt., 2/XI/1993, O. Yano et al. 21126p.p. (SP 274120); Campo Grande, reserva Biológica, Univ. Federal de MS, sobre húmus na mata úmida, 8/IV/1998, O. Yano \& J. Xavier 25264 (SP 322077).

Cresce associada a Riccardia cataractarum (Spruce) Hell.

\section{MARCHANTIACEAE}

\section{*Dumortiera hirsuta (Sw.) Nees}

Material examinado: BRASIL. Mato Grosso do Sul: munic. de Inocência, ca. $51^{\circ} 48^{\prime} \mathrm{W}, 19^{\circ} 46^{\prime} \mathrm{S}$, ca. 400m alt., rod. MS São Pedro-Inocência, ca. $17 \mathrm{~km}$ de Inocência, cerrado, barranco úmido, 11/XI/1995, M.R. Pietrobom-Silva \& M. de Lucca Jr. 2476 (SP 322772).

\section{Marchantia papillata Raddi}

Material examinado: BRASIL. Mato Grosso do Sul: munic. de Bonito, ca. 56 $28^{\circ} \mathrm{W}, 21^{\circ} 8^{\prime} \mathrm{S}$, ca. $300 \mathrm{~m}$ alt., MS-178 Bonito-Bodoquena, Rio Formoso, cachoeira próximo da estrada, em barranco úmido, 4/VIII/1994, C.E. Rodrigues Jr. \& M.R. PietrobomSilva 786p.p. (SP 322861).

Cresce associada a Barbula indica (Hook.) Spreng.

\section{PALLAVICINIACEAE}

*Pallavicinia lyellii (Hook.) S.F. Gray

Material examinado: BRASIL. Mato Grosso do Sul: munic. de Indaiá do Sul, ca. 52 $23^{\prime} \mathrm{W}, 18^{\circ} 57^{\prime} \mathrm{S}$, ca. $500 \mathrm{~m}$ alt., cachoeira aos fundos da cidade, capões de cerrado, sobre tronco de árvore, 18/II/1996, M.R. Pietrobom-Silva et al. 2940 (SP 322795).

\section{PLAGIOCHILACEAE}

*Plagiochila disticha (Lehm. \& Lindenb.) Mont.

Material examinado: BRASIL. Mato Grosso do Sul: munic. de Costa Rica, ca. 53 $3^{\circ} 13^{\prime} \mathrm{W}-18^{\circ} 47^{\prime} \mathrm{S}$, ca. $450 \mathrm{~m}$ alt., Ribeirão Sucuruí, aos fundos da cidade de Costa Rica, sobre tronco em decomposição, 20/II/1996, M.R. Pietrobom-Silva et al. 2986p.p. (SP 322820); munic. de Selviria, Córrego da Véstia, base do tronco, 5/XI/1985, O. Yano \& M.R.P Noronha 9592 (SP 206297).

Cresce associada a Racopilum tomentosum (Hedw.) Brid.

Plagiochila martiana (Nees) Lindenb.

Material examinado: BRASIL. Mato Grosso do Sul: munic. de Aparecida do Taboado, ca. $51^{\circ} 05^{\prime} \mathrm{W}$, $20^{\circ} 06^{\prime} \mathrm{S}$, ca. $300 \mathrm{~m}$ alt., BR-158 Ap. do TaboadoParanaíba, ca. 20km de Ap. do Taboado, capões de cerradão, sobre tronco de peroba, 17/II/1996, M.R. Pietrobom-Silva et al. 2835 (SP 322779); munic. de Indaiá do Sul, ca. $52^{\circ} 23^{\prime} \mathrm{W}, 18^{\circ} 57^{\prime} \mathrm{S}$, ca. $500 \mathrm{~m}$ alt., cachoeira aos fundos da cidade, capões de cerrado, sobre tronco em decomposição, 18/II/1996, M.R. Pietrobom-Silva et al. 2946, 2947p.p. (SP 322801; SP 322802); munic. de Dourados, Faz. Sr. Antonio Tonani, sobre tronco de árvore, 25/I/1979, A.I. Milanez 
s.n. (SP 133219); munic. de Três Lagoas, reserva Agroflorestal, sobre tronco de árvore na mata, $\pm 230 \mathrm{~m}$ alt., 12/IX/1992, O. Yano \& M.P. Marcelli 17125 (SP 2442264); munic. de Selviria, Faz. do Cacildo, $\pm 40 \mathrm{~km}$ de Ilha Solteira em direção a Três Lagoas, base do tronco na região pantanosa perto do cerradão, 24/X/1984, O. Yano \& N. Taroda 9291 (SP 191912); idem, Córrego da Véstia, base do tronco, 5/XI/1985, O. Yano \& M.R.P. Noronha 9584, 9595, 9603 (SP 206289; SP 206300; SP 206308).

Cresce associada a Chyrso-hypnum diminutium (Hampe) W.R. Buck.

\section{RADULACEAE}

Radula macrostachya Lindenb. \& Gottsche

Material examinado: BRASIL. Mato Grosso do Sul: munic. de Costa Rica, ca. 53 ${ }^{\circ} 13^{\prime} \mathrm{W}, 18^{\circ} 47^{\prime} \mathrm{S}$, ca. $450 \mathrm{~m}$ alt., Ribeirão Sucuruí, aos fundos da cidade de Costa Rica, sobre tronco em decomposição, 20/II/1996, M.R. Pietrobom-Silva et al. 2983p.p. (SP 322817).

Cresce associada a Calymperes afzelii Sw.

\section{RICCIACEAE}

Riccia sp.

Material examinado: BRASIL. Mato Grosso do Sul: munic. de Corumbá, ca. $12 \mathrm{~km}$ SE da cidade, solo rochoso, 2/XII/1979, D.M. Vital 8602, 8603 (SP 133314; SP 133315); munic. de Aquidauana, sobre rochas areníticas, 3/XII/1979, D.M. Vital 8611 (SP 133317).

\section{BRYOPHYTA}

\section{BARTRAMIACEAE}

*Philonotis hastata (Duby) Wijk \& Margad.

Material examinado: BRASIL. Mato Grosso do Sul: munic. de Costa Rica, ca. 53 ${ }^{\circ} 13^{\prime} \mathrm{W}, 18^{\circ} 47^{\prime} \mathrm{S}$, povoado Laje, ca. 550m alt., MS-306 Cantina Costa Rica-Capela, cachoeira do Rio Laje, capões de cerrado, sobre barranco rochoso, 20/II/1996, M.R. Pietrobom-Silva et al. 2976p.p. (SP 322808); munic. de Rio Verde de Mato Grosso, ca. 54 $53^{\circ}$ W, $18^{\circ} 55^{\prime}$ 'S, ca. 300m alt., Serra Pimenteira, cachoeira Babaçu, Faz. Quartel, terrestre junto do riacho, 6/II/1994, M.R. Pietrobom-Silva \& C.E. Rodrigues Jr. 1287 (SP 322866).

Cresce associada a Vesicularia vesicularis (Schwägr.) Broth.
*Philonotis uncinata (Schwägr.) Brid.

Material examinado: BRASIL. Mato Grosso do Sul: munic. de Corumbá, solo rochoso, canga, 2/XII/1979, D.M. Vital 8604 (SP 147893); idem, Morro Tromba dos Macacos, paredão escorrendo água da cachoeira, $\pm 250 \mathrm{~m}$ alt., 2/XI/1993, O. Yano et al. 21121 (SP 274115).

\section{BRACHYTHECIACEAE}

*Brachythecium ruderale (Brid.) W.R. Buck

Material examinado: BRASIL. Mato Grosso do Sul: munic. de Rochedo, bordos de um riacho (19.57'S, 5507'W), 25/I/1979, D.M. Vital 8433 (SP 147505); Selviria, Estação Experimental da UNESP, margem de uma lagoa perto do cerrado, 7/XI/1985, O. Yano \& M.R. Pereira-Noronha 9727 (SP 206432).

*Rhynchostegium scariosum (Tayl.) A. Jaeger

Material examinado: BRASIL. Mato Grosso do Sul: munic. de Alto Taquari, ca. $53^{\circ} 17^{\prime} \mathrm{W}, 17^{\circ} 50^{\prime} \mathrm{S}$, ca. $600-650 \mathrm{~m}$ alt., ca. $25 \mathrm{~km}$ à sudeste da cidade, córrego da Laje, paredão rochoso úmido, 21/II/1996, M.R. Pietrobom-Silva et al. 3015 (SP 322789).

\section{BRYACEAE}

*Brachymenium radiculosum (Schwägr.) Hampe

Material examinado: BRASIL. Mato Grosso do Sul: munic. de Corumbá, solo rochoso, canga, 2/XII/1979, D.M. Vital 8601 (SP 147892).

\section{*Bryum attenense Williams}

Material examinado: BRASIL. Mato Grosso do Sul: Corumbá, barranco úmido perto do canal Tameiro em frente a cidade de Quijarro, 25/I/1979, O. Yano 1350 (SP 147480); idem, sobre tronco de Bacurina, praça da Independência, 28///1979, D.M. Vital 8450 (SP 147521).

\section{*Rosulabryum billardierei (Schwägr.) Spence}

Material examinado: BRASIL. Mato Grosso do Sul: munic. de Costa Rica, ca. 53 ${ }^{\circ} 13^{\prime} \mathrm{W}, 18^{\circ} 47^{\prime} \mathrm{S}$, povoado Laje, ca. 550m alt., MS-306 Cantina Costa Rica-Capela, cachoeira do Rio Laje, capões de cerrado, sobre barranco rochoso, 20/II/1996, M.R. Pietrobom-Silva et al. 2977 (SP 322809); munic. de Rio Verde de Mato Grosso, ca. 545' W, 1855'S, ca. $400 \mathrm{~m}$ alt., Serra Pimenteira, cachoeira do Cervo, em barraco úmido, 22/II/1994, C.E. Rodrigues Jr. \& M.R. Pietrobom-Silva 737 (SP 322858). 


\section{CALYMPERACEAE}

*Calymperes afzelii $\mathrm{Sw}$.

Material examinado: BRASIL. Mato Grosso do

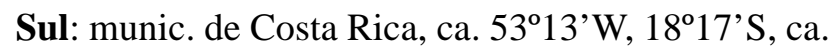
$450 \mathrm{~m}$ alt., Ribeirão Sucuruí, aos fundos da cidade de Costa Rica, sobre tronco em decomposição, 20/II/1996, M.R. Pietrobom-Silva et al. 2983p.p. (SP 322817).

Cresce associada a Cheilolejeunea rigidula (Mont.) R.M. Schust. e Radula macrostachya Lindenb. \& Gottsche.

*Syrrhopodom ligulatus Mont.

Material examinado: BRASIL. Mato Grosso do Sul: munic. de Bonito, base de um tronco de árvore, 28/XI/1979, D.M. Vital 8593 (SP 147887); munic. de Selviria, Faz. Estação Experimental da UNESP, $\pm 18 \mathrm{~km}$ da Ilha Solteira em direção a Ap. do Tabuado, base do tronco no cerradão, 22/X/1984, O. Yano \& J. Semir 9256 (SP 191877); idem, Faz. Agropecuária Santa Maria, base do tronco no cerradão, 7/XI/1985, O. Yano \& M.R. Pereira-Noronha 9746 (SP 206450).

*Syrrhopodon parasiticus (Brid.) Besch.

Material examinado: BRASIL. Mato Grosso do Sul: munic. de Corumbá, Morro Tromba dos Macacos, sobre tronco de Palmae na mata, $\pm 230 \mathrm{~m}$ alt., 2/XI/1993, O. Yano et al. 21105 (SP 274100).

\section{CRYPHAEACEAE}

*Schoenobryum concavifolium (Griff.) Gangulee

Material examinado: BRASIL. Mato Grosso do Sul: munic. de Rio Brilhante, sobre tronco de Sapindaceae no cerrado, 27/XI/1979, D.M. Vital 8563 (SP 147867).

\section{DICRANACEAE}

\section{Campylopus carolinae Grout}

Material examinado: BRASIL. Mato Grosso do Sul: munic. de Costa Rica, ca. $53^{\circ} 13^{\prime} \mathrm{W}, 18^{\circ} 47^{\prime} \mathrm{S}$, povoado Laje, ca. 550m alt., MS-306 Cantina Costa Rica-Capela, cachoeira do Rio Laje, capões de cerrado, sobre paredão rochoso, 20/II/1996, M.R. Pietrobom-Silva et al. 2975 (SP 322807).

*Campylopus savannarum (Müll. Hal.) Mitt.

Material examinado: BRASIL. Mato Grosso do Sul: munic. de Rio Verde de Mato Grosso, ca. 54 $53^{\circ}$ 'W, $18^{\circ} 55^{\prime}$ 'S, ca. $400 \mathrm{~m}$ alt., ca. 30km da cidade, Faz. Mirante, rod. Sete Quedas-Rio Negro, sobre rocha, 7/IX/1993, M.R. Pietrobom-Silva 1140 (SP 322864).

*Dicranella hilariana (Mont.) Mitt.

Material examinado: BRASIL. Mato Grosso do Sul: munic. de Ribas do Rio Pardo, reflorestadora Estância S.A., regos de drenagem perto do rio Bota, 25/I/1979, O. Yano 1341 (SP 147473).

*Microcampylopus kouroussensis (Ren. \& Par.) Rich. \& Clear

Material examinado: BRASIL. Mato Grosso do Sul: munic. de Rio Brilhante, solo, do cerrado, 27/XI/1979, D.M. Vital 8565 (SP 147868); idem, 3/XII/1979, D.M. Vital 8614 (SP 147901).

\section{ENTODONTACEAE}

Entodon beyrichii (Schwägr.) Müll. Hal.

Material examinado: BRASIL. Mato Grosso do Sul: munic. de Jardim, sobre pau podre, 28/XI/1979, D.M. Vital 8577 (SP 147878).

*Entodon macropodus (Hedw.) Müll. Hal.

Material examinado: BRASIL. Mato Grosso do Sul: munic. de Rio Verde de Mato Grosso, ca. 54 $53^{\circ}$ 'W, $18^{\circ} 55^{\prime} \mathrm{S}$, ca. $380 \mathrm{~m}$ alt., Serra da Alegria, ca. $18 \mathrm{~km}$ da BR-169, sobre tronco de árvore, 24/II/1994, M.R. Pietrobom-Silva \& C.E. Rodrigures Jr. 1309 (SP 322868).

*Erythrodontium squarrosum (Müll. Hal.) Par.

Material examinado: BRASIL. Mato Grosso do Sul: munic. de Rio Verde de Mato Grosso, ca. $54^{\circ} 53^{\prime} \mathrm{W}, 18^{\circ} 55^{\prime}$ 'S, ca. $400 \mathrm{~m}$ alt., rod. Sete QuedasRio Negro, ca. $30 \mathrm{~km}$ de cidade, Faz. Mirante, cachoeira do Cervo, Serra Pimenteira, barranco úmido, 4/VIII/1994, C.E. Rodrigues Jr. \& M.R. Pietrobom-Silva 738 (SP 322859); munic. de Bonito, ca. $4 \mathrm{~km} \mathrm{~S}$ da cidade, sobre tronco de árvore isolada no cerrado, 28/XI/1979, D.M. Vital 8586 p.p. (SP 133308).

Cresce associada a Frullania gibbosa Nees.

\section{ERPODIACEAE}

Aulacopilum glaucum Wils.

Material examinado: BRASIL. Mato Grosso do Sul: Campo Grande, sobre tronco de Michelia sp., rua Rio Branco (20²7'S, 54²38'W), 27/I/1979, D.M. Vital 8448 (SP147519). 
Erpodium beccarii Müll. Hal. ex Vent.

Material examinado: BRASIL. Mato Grosso do Sul: Corumbá, morro do Urucum, sobre tronco de Ficus próximo a estrada para as minas, 29/I/1979, O. Yano 1358 (SP 147488); Campo Grande, sobre tronco de Caesalpinia peltophoroide, 24/I/1979, D.M. Vital 8413 (SP 147494); munic. de Corumbá, Morro Tromba dos Macacos, sobre tronco de Bocaiúva, $\pm 210 \mathrm{~m}$ alt., 2/XI/1993, O. Yano et al. 21063, 21074 (SP 274059; SP 274070).

Erpodium coronatum (Hook. \& Wils.) Mitt.

Material examinado: BRASIL. Mato Grosso do Sul: munic. de Selviria, Faz. Estação Experimental da UNESP, $\pm 18 \mathrm{~km}$ da Ilha Solteira, em direção a Aparecida do Taboado, sobre galhos de arbusto no cerradão, 22/X/1984, O. Yano \& J. Semir 9262 (SP 191883); idem, $\pm 40 \mathrm{~km}$ da Ilha Solteira em direção a Três Lagoas, sobre galhos de árvore no cerradão, 23/X/1984, O. Yano \& N. Taroda 9273, 9277 (SP 191894; SP 191898); idem, 25/X/1984, O. Yano \& N. Taroda 9300 (SP 191920); idem, Córrego da Véstia, sobre tronco, 5/XI/1985, O. Yano \& M.R. Pereira-Noronha 9606, 9612 (SP 206311; SP 206317); munic. de Ribas do Rio Pardo, sobre tronco de Terminalia catapa, 25/I/1979, O. Yano 1335p.p., 1377 (SP 133224; SP 147470); idem, sobre tronco de Pinus, perto da rua J.C. Garcia, 25/I/1979, O. Yano 1339 (SP 147472); munic. de Corumbá $\left(19^{\circ} 12^{\prime} \mathrm{S}, 5^{\circ} 31^{\prime} \mathrm{W}\right)$, sobre tronco de bacuri, $\pm 15 \mathrm{~m}$ alt., 29/I/1979, O. Yano 1352 (SP 147482); idem, Morro do Urucum (19 $12^{\circ}$ 'S, $\left.57^{\circ} 38^{\prime} \mathrm{W}\right)$, sobre tronco de Ficus próximo a estrada para minas, 29/I/1979, O. Yano 1356 (SP 147486); Campo Grande (20²7'S, 54³7' W), sobre tronco de palmeira, 22/I/1979, D.M. Vital 8408 (SP 147491); idem, sobre tronco de Delonix, 24/I/1979, D.M. Vital 8412 (SP 147493); munic. de Ladário, sobre tronco de bacuri, 29/I/1979, D.M. Vital 8452 (SP 147523); munic. de Rochedo, sobre tronco de Helicteres sp., em cerrado, 25/I/1979, D.M. Vital 8425, 8426 (SP 147500; SP 147501); munic. de Rio Brilhante, sobre tronco de Sapotaceae, em cerrado, 27/XI/1979, D.M. Vital 8562 (SP 147866); munic. de Bonito, sobre tronco de Magonia no cerrado, 28/XI/1979, D.M. Vital 8588 (SP 147884); munic. de Aquidauana, ca. $30 \mathrm{~km}$ W-NW, sobre tronco de árvore no cerradão, 3/XII/1979, D.M. Vital 8607 (SP 147895); munic. de Corumbá, Morro Tromba dos Macacos, sobre tronco de Mangifera, $\pm 210 \mathrm{~m}$ alt., 2/XI/1993, O. Yano et al. 21032 (SP 274030); idem, sobre tronco de Bocaiúva,
2/XI/1993, O. Yano et al. 21053 (SP 274050); munic. de Selviria, Estação Experimental da UNESP, sobre tronco, cerradão, 7/XI/1985, O. Yano \& M.R. PereiraNoronha 9714, 9724 (SP 206419; SP 206429); Campo Grande, campus da Univ. Federal de MS, sobre tronco de árvore, 8/IV/1998, O. Yano \& J. Xavier 25223, 25235, 25236 (SP 322040; SP 322050; SP 322051).

\section{Erpodium glazioui Hampe}

Material examinado: BRASIL. Mato Grosso do Sul: Dourados, 1/II/1979, M.T. Paiva Azevedo s.n. (SP 147532); Campo Grande, sobre tronco de Inga sp., na praça de República, 27/I/1979, D.M. Vital 8446p.p. (SP 133239); munic. de Ribas do Rio Pardo, sobre tronco de árvore na rua Filinto Müller, 25/I/1979, O. Yano 1334 (SP 147468); idem, sobre tronco de Terminalia catapa, 25/I/1979, O. Yano 1338 (SP 147471); Campo Grande $\left(20^{\circ} 27^{\prime} \mathrm{S}\right.$, $54^{\circ} 37^{\prime} \mathrm{W}$ ), sobre tronco de Yuca, na praça Afonso Pena, 22/I/1979, D.M. Vital 8407 (SP 147490); Rio Brilhante, na praça Boa Ventura, sobre tronco de Delonix sp., 27/XI/1979, D.M. Vital 8567 (SP 147869); Maracaju, sobre tronco de Arecastrum sp., 27/XI/1979, D.M. Vital 8569 (SP 149871); munic. de Corumbá, sobre tronco fino de árvore, 30/XI/1979, D.M. Vital 8598 (SP 147889); Miranda, sobre tronco de Mangifera indica, 3/XII/1979, D.M. Vital 8605 (SP 147894); Três Lagoas, praça perto da rua principal de usina, sobre tronco de Terminalia sp., 25/VIII/1983, O. Yano \& R.C. Compagnoli 8297 (SP 189733); munic. de Corumbá, Morro Tromba dos Macacos, sobre tronco de Mangifera perto do riacho, $\pm 210 \mathrm{~m}$ alt., 2/XI/1993, O. Yano et al. 2103 (SP 274028); idem, sobre tronco de árvore, 2/XI/1993, O. Yano et al. 21065 (SP 274061); Campo Grande, Campus da Univ. Federal de MS, sobre tronco de árvore, 8/IV/1998, O. Yano \& J. Xavier 25225, 25233 (SP 322042; SP 322048).

Cresce associada a Fabronia ciliaris var. polycarpa (Hook.) W.R. Buck, Frullania ericoides (Nees) Mont.

\section{Erpodium pringlei Britt.}

Material examinado: BRASIL. Mato Grosso do Sul: munic. de Campo Grande, sobre tronco de Spathodea sp., 27/I/1979, D.M. Vital 8438 (SP 147510); idem, sobre tronco de Michelia sp., 27/I/1979, D.M. Vital 8447 (SP 147518); munic. de Guia Lopes da Laguna, ca. 40km E da cidade, sobre tronco de Bombacaceae em cerradão, 27/XI/1979, D.M. Vital 8571, 8574 (SP 147873; SP 147875). 


\section{FABRONIACEAE}

*Dimerodontium mendozense Mitt.

Material examinado: BRASIL. Mato Grosso do Sul: munic. de Rochedo, sobre tronco de árvore, ao longo do rio Aquidauana ( $\left.20^{\circ} 08^{\prime} \mathrm{S}, 55^{\circ} 04^{\prime} \mathrm{W}\right), 25 / \mathrm{I} / 1979$, D.M. Vital 8419p.p. (SP 133230).

Cresce associada a Frullania inflata Gottsche e Lejeunea trinitensis Lindenb.

Fabronia ciliaris (Brid.) Brid. var. polycarpa (Hook.) W.R. Buck

Material examinado: BRASIL. Mato Grosso do Sul: Campo Grande, praça próximo ao QG do exército, sobre tronco de Inga sp., 31/I/1979, D.M. Vital 8461p.p. (SP 133241); idem, na praça de República, 27/I/1979, D.M. Vital 8446p.p. (SP 133239); munic. de Ribas do Rio Pardo, base de árvore, perto da linha de ferro, paralela a rua J.C. Garcia, 25/I/1979, O. Yano 1333 (SP 147467); idem, sobre tronco de Terminalia catapa, 25/I/1979, O. Yano 1336, 1342 (SP 147469; SP 147474); munic. de Corumbá, morro do Urucum $\left(19^{\circ} 12^{\prime} \mathrm{S}\right.$, $\left.57^{\circ} 38^{\prime} \mathrm{W}\right)$, sobre tronco de Urucum, $\pm 10 \mathrm{~m}$ alt., 29/I/1979, O. Yano 1353 (SP 147483); idem, sobre tronco de Ficus próximo a estrada para as minas, 29/I/1979, O. Yano 1357 (SP 147487); Campo Grande, sobre tronco de Mangifera indica, 24/I/1979, D.M. Vital 8411 (SP 147492); munic. de Corumbá, Morro Tromba dos Macacos, sobre tronco de Leguminosae, $\pm 210 \mathrm{~m}$ alt., 2/XI/1993, O. Yano et al. 21048 (SP 274045); idem sobre estipe de Bocaiúva, $\pm 210 \mathrm{~m}$ alt., 2/XI/1993, O. Yano et al. 21079 (SP 274074); munic. de Selviria, Faz. do Cacildo, $\pm 40 \mathrm{~km}$ da Ilha Solteira em direção a Três Lagoas, nos ritidomas do tronco no cerradão, 25/X/1984, O. Yano \& N. Taroda 9297 (SP 191918); Campo Grande, no campus da Univ. Federal de MS, sobre tronco de árvore, 8/IV/1998, O. Yano \& J. Xavier 25224, 25227 (SP 322041; SP 322044).

Cresce associada a Erpodium glazioui Hampe e Lejeunea laetevirens Nees \& Mont.

*Fabronia macroblepharis Schwägr.

Material examinado: BRASIL. Mato Grosso do Sul: munic. de Brasilândia, Lagoa Machado, ca. $52^{\circ} 07^{\prime} 54^{\prime \prime} \mathrm{W}, 21^{\circ} 43^{\prime} 51^{\prime \prime} \mathrm{S}$, ca. $7 \mathrm{~km}$ da sede pela trilha turística, sobre tronco de bacuri, próximo do varjão, 24/IX/1996, M.R. Pietrobom-Silva 3529 (SP 322825); idem, sobre tronco de bacuri, 25/IX/1996, M.R.
Pietrobom-Silva 3543 (SP 322826); munic. de Corumbá, $2 \mathrm{~km}$ antes do limite do Pantanal, sobre tronco de bacuri, $\pm 15 \mathrm{~m}$ alt., 29/I/1979, O. Yano 1351 (SP 147481); idem, Centro-Oeste, Faz. Campo Dora, 11/IX/1988, V.I. Pott et al. 667 (SP 280984).

\section{FISSIDENTACEAE}

*Fissidens elegans Brid.

Material examinado: BRASIL. Mato Grosso do Sul: munic. de Corumbá, Morro Tromba dos Macacos, sobre minério de ferro na mata, $\pm 230 \mathrm{~m}$ alt., $2 / \mathrm{XI} / 1993$, O. Yano et al. 21100 (SP 274095).

*Fissidens flaccidus Mitt.

Material examinado: BRASIL. Mato Grosso do Sul: munic. de Ribas do Rio Pardo, $\pm 16 \mathrm{~km}$ antes da cidade, margem do rio, 25/I/1979, O. Yano 1349 (SP 147479); munic. de Corumbá, Morro Tromba dos Macacos, solo arenoso da mata, $\pm 210 \mathrm{~m}$ alt., 2/XI/1993, O. Yano et al. 21084 (SP 274078).

*Fissidens intramarginatus (Hampe) Mitt.

Material examinado: BRASIL. Mato Grosso do Sul: munic. de Costa Rica, ca. 53 $3^{\circ} 13^{\prime} \mathrm{W}, 18^{\circ} 47^{\prime} \mathrm{S}$, povoado Laje, ca. 550m alt., MS-306 Cantina Costa Rica-Capela, cachoeira do Rio Laje, capões de cerrado, sobre barranco, 20/II/1996, M.R. PietrobomSilva et al. 2977a (SP 322810); munic. de Corumbá, Morro Tromba dos Macacos, paredão escorrendo água, 2/XI/1993, O. Yano et al. 21134p.p. (SP 274128).

Cresce associada a Phaeoceros laevis (L.) Prosk.

*Fissidens microcladus Thwait. \& Mitt.

Material examinado: BRASIL. Mato Grosso do Sul: munic. de Aquidauana, sobre rochas areníticas, na encosta da serra de Santa Bárbara, 3/XII/1979, D.M. Vital 8610, R.A. Pursell (SP 147898).

*Fissidens rigidulus Hook. \& Wils.

Material examinado: BRASIL. Mato Grosso do Sul: munic. de Corumbá, Morro Tromba dos Macacos, sobre pedra no riacho, $\pm 230 \mathrm{~m}$ alt., 2/XI/1993, O. Yano et al. 21108 (SP 274103).

\section{*Fissidens submarginatus Bruch in C. Krauss}

Material examinado: BRASIL. Mato Grosso do Sul: munic. de Rochedo, sobre cupinzeiro, em cerrado, 25/I/1979, D.M. Vital 8428 (SP 147503). 
Fissidens termitarum (Hook.) Pursell

Material examinado: BRASIL. Mato Grosso do Sul: munic. de Rochedo ( $\left.20^{\circ} 08^{\prime} \mathrm{S}, 55^{\circ} 04^{\prime} \mathrm{W}\right)$, sobre tronco de Copaifera sp. ao longo do rio Aquidauana, 25/I/1979, D.M. Vital 8418, R.A. Pursell (SP 147499); munic. de Aquidauana, sobre termitério na encosta da serra Santa Bárbara, 3/XII/1979, D.M. Vital 8613, R.A. Pursell (SP 147900).

*Fissidens zollingeri Mont.

Material examinado: BRASIL. Mato Grosso do Sul: munic. de Corumbá, Morro Tromba dos Macacos, solo terroso perto do riacho, $\pm 230 \mathrm{~m}$ alt., $2 / \mathrm{XI} / 1993$, O. Yano et al. 21093 (SP 274088).

\section{FUNARIACEAE}

*Funaria obtusata Schimp. ex Müll. Hal.

Material examinado: BRASIL. Mato Grosso do Sul: munic. de Corumbá, parede úmida da casa, 1/XII/1979, D.M. Vital 8600 (SP 147891).

\section{HELICOPHYLLACEAE}

\section{Helicophyllum torquatum (Hook.) Brid.}

Material examinado: BRASIL. Mato Grosso do Sul: munic. de Bonito, ca. 22km N-NW da cidade, sobre tronco na encosta do morro, 28/XI/1979, D.M. Vital 8596p.p. (SP 133312); munic. de Corumbá, Morro do Urucum, $\left(19^{\circ} 12^{\prime} \mathrm{S}, 57^{\circ} 38^{\prime} \mathrm{W}\right)$, sobre tronco de árvore, 29/I/1979, O. Yano 1355 (SP 147485); Campo Grande, sobre tronco de Ficus bejaminiana na praça Afonso Pena, 26/I/1979, D.M. Vital 8406 (SP 147489); idem, Faz. Canaan, ca. $18 \mathrm{~km}$ W de Campo Grande, sobre tronco podre, 24/I/1979, D.M. Vital 8416 (SP 147497); munic. de Rochedo, sobre tronco de Sapindaceae, 25/I/1979, D.M. Vital 8434 (SP 147506); munic. de Selviria, Córrego da Véstia, sobre tronco, 5/XI/1985, O. Yano \& M.R.P. Noronha 9598 (SP 206303); munic. de Corumbá, Morro Tromba dos Macacos, sobre tronco de Bocaiúva, $\pm 210 \mathrm{~m}$ alt., 2/XI/1993, O. Yano et al. 21052 (SP 274049).

Cresce associada a Schiffneriolejeunea polycarpa (Nees) Gradst.

\section{HOOKERIACEAE}

Callicostella pallida (Hornsch.) Aongstr.

Material examinado: BRASIL. Mato Grosso do Sul: munic. de Ribas do Rio Pardo, $\pm 71 \mathrm{~km}$ de Campo Grande, barranco perto do rio, 25/I/1979, O. Yano
1326 (SP 147463); idem, $\pm 16 \mathrm{~km}$ antes da cidade, margem do rio, 25/I/1970, O. Yano 1348 (SP 147478); munic. de Corumbá, Morro Tromba dos Macacos, sobre raízes de arbusto no riacho, $\pm 210 \mathrm{~m}$ alt., 2/XI/1993, $O$. Yano et al. 21041 (SP 274039); idem, sobre pedra no riacho, $\pm 210 \mathrm{~m}$ alt., 2/XI/1993, O. Yano et al. 21110 (SP 274105); idem, paredão escorrendo água, $\pm 250 \mathrm{~m}$ alt., 2/XI/1993, O. Yano et al. 21120 (SP 274114); Campo Grande, reserva Biológica, Univ. Federal de MS, sobre raízes, mata úmida, 8/IV/1998, O. Yano \& J. Xavier 25260, 25261 (SP 322073; SP 322074).

\section{HYPNACEAE}

Chryso-hypnum diminutivum (Hampe) W.R. Buck

Material examinado: BRASIL. Mato Grosso do Sul: munic. de Aparecida do Taboado, ca. $51^{\circ} 05^{\prime} \mathrm{W}$, $20^{\circ} 06^{\prime} \mathrm{S}$, ca. $300 \mathrm{~m}$ alt., rod. BR-158, sobre tronco em decomposição, 17/II/1996, M.R. Pietrobom-Silva et al. 2830p.p., 2833 (SP 322774; SP 322777); munic. de Paranaíba, ca. 51 $32^{\circ}$ 'W, $1^{\circ} 37^{\prime}$ 'S, BR-158 ParanaíbaCassilândia, ca. $5 \mathrm{~km}$ do Porto Raimundo, capões de mata seca, sobre tronco em decomposição, 17/II/1996, M.R. Pietrobom-Silva et al. 2876p.p. (SP 322781); munic. de Indaiá do Sul, ca. $52^{\circ} 23^{\prime} \mathrm{W}, 18^{\circ} 57^{\prime} \mathrm{S}$, ca. $500 \mathrm{~m}$ alt., cachoeira aos fundos da cidade, capões de cerrado, sobre tronco em decomposição, 18/II/1996, M.R. Pietrobom-Silva et al. 2947p.p. (SP 322802); munic. de Costa Rica, ca. $53^{\circ} 13^{\prime} \mathrm{W}, 18^{\circ} 47^{\prime} \mathrm{S}$, ca. $450 \mathrm{~m}$ alt., Ribeirão Sucuri, aos fundos da cidade de Costa Rica, sobre tronco em decomposição, 20/II/1996, M.R. Pietrobom-Silva et al. 2988p.p. (SP 322822); munic. de Alto Taquari, ca. $53^{\circ} 17^{\prime} \mathrm{W}, 17^{\circ} 51^{\prime} \mathrm{S}$, ca. $900 \mathrm{~m}$ alt., MT-100 Alto Taquari-Alto Araguaia, ca. $6 \mathrm{~km}$ da cidade, Faz. Bambuzal, barranco úmido, 21/II/1994, C.E. Rodrigues Jr. \& M.R. Pietrobom-Silva 694 (SP 322832); idem, ca. $600-650 \mathrm{~m}$ alt., ca. $25 \mathrm{~km}$ a sudeste da cidade, córrego da Laje, sobre tronco em decomposição, 21/II/1996, M.R. Pietrobom-Silva et al. 3018 (SP 322842); munic. de Selviria, Faz. Estação Experimental da UNESP, $\pm 18 \mathrm{~km}$ de Ilha Solteira em direção a Ap. do Tabuado, sobre tronco no cerradão, 22/X/1984 O. Yano \& J. Semir 9261 (SP 191882); Campo Grande, reserva Biológica da Univ. Federal de MS, sobre tronco de arbusto na mata úmida, 8/IV/1998 O. Yano \& J. Xavier 25249 (SP 322062); idem, base do tronco de leiteira, mata úmida, 8/IV/1998 O. Yano \& J. Xavier 25291 (SP 322104).

Cresce associada a Cyrto-hypnum involvens (Mitt.) W.R. Buck \& H.A. Crum, Lejeunea glaucescens Gottsche, Plagiochila martiana (Nees) 
Lindenb. e Vesicularia vesicularis (Schwägr.) Broth.

Chryso-hypnum elegantulum (Hook.) Hampe

Material examinado: BRASIL. Mato Grosso do Sul: munic. de Selviria, ca. $51^{\circ} 25^{\prime} \mathrm{W}, 2^{\circ} 23^{\prime}$ 'S, ca. 300m alt., rod. MS Selviria-São Pedro, ca. $41 \mathrm{~km}$ da cidade de Selviria, cerrado, 11/XI/1995, M.R. Pietrobom-Silva \& M. de Lucca Jr. 2453 (SP 322771); munic. de Indaiá do Sul, ca. $52^{\circ} 23^{\prime} \mathrm{W}, 18^{\circ} 57^{\prime} \mathrm{S}$, ca. $500 \mathrm{~m}$ alt., cachoeira aos fundos da cidade, capões de cerrado, sobre tronco de árvore, 18/II/1996, M.R. Pietrobom-Silva et al. 2944p.p. (SP 322799); munic. de Três Lagoas, base do tronco de árvore, $\pm 230 \mathrm{~m}$ alt., 12/IX/1992, O. Yano \& M.P. Marcelli 17023 (SP 242168).

Cresce associada a Papillaria nigrescens (Hedw.) A. Jaeger.

\section{*Cyclodictyon albicans (Hedw.) O. Kuntze}

Material examinado: BRASIL. Mato Grosso do Sul: munic. de Ribas do Rio Pardo, $\pm 16 \mathrm{~km}$ da cidade, margem do rio, 25/I/1979, O. Yano 1347 (SP 133227); munic. de Corumbá, Morro Tromba dos Macacos, sobre pedra no riacho na mata, $\pm 230 \mathrm{~m}$ alt., $2 / \mathrm{XI} / 1993$, O. Yano et al. 21097 (SP 274092); idem, pendente em raízes como cortina na cachoeira, $\pm 250 \mathrm{~m}$ alt., 2/XI/1993, O. Yano et al. 21133 (SP 274127).

\section{*Cyclodictyon varians (Sull.) O. Kuntze}

Material examinado: BRASIL. Mato Grosso do Sul: munic. de Corumbá, Morro Tromba dos Macacos, pendentes em raízes como cortina na cachoeira, $\pm 250 \mathrm{~m}$ alt., 2/XI/1993, O. Yano et al. 21125 (SP 274119).

*Ectropothecium leptochaeton (Schwägr.) W.R. Buck

Material examinado: BRASIL. Mato Grosso do Sul: munic. de Indaiá do Sul, ca. $52^{\circ} 23^{\prime} \mathrm{W}, 18^{\circ} 57^{\prime} \mathrm{S}$, ca. $500 \mathrm{~m}$ alt., cachoeira aos fundos da cidade, capões de cerrado, sobre tronco de árvore, 18/II/1996, M.R. Pietrobom-Silva et al. 2942 (SP 322797).

Isopterygium tenerum (Sw.) Mitt.

Material examinado: BRASIL. Mato Grosso do Sul: munic. de Rio Verde de Mato Grosso, ca. 54 $53^{\circ}$ 'W, $18^{\circ} 55^{\prime}$ 'S, ca. $400 \mathrm{~m}$ alt., rod. Sete Quedas-Rio Negro, ca. $30 \mathrm{~km}$ da cidade, Fazenda Mirante, cachoeira do Cervo, sobre árvore, 7/IX/1993, C.E. Rodrigues Jr. \& M.R. Pietrobom-Silva 662 (SP 322850); munic. de Rochedo, perto do rio Sabão, 25/I/1979, R.G. da Silva s.n. (SP 147453); munic. de Dourados, $2 \mathrm{~km}$ além do rio Vacaria na estrada Campo Grande-Dourados, sobre tronco de palmeira, 25/I/1979, A.I. Milanez s.n.
(SP 147458); idem, Faz. Sr. Antonio Tonani, sobre pau podre, 25/I/1979, A.I. Milanez s.n. (SP 147459); munic. de Jardim, sobre pau podre, 28/XI/1979, D.M. Vital 858 (SP 147880); munic. de Corumbá, Morro Tromba dos Macacos, sobre tronco podre no riacho, $\pm 230 \mathrm{~m}$ alt., 2/XI/1993, O. Yano et al. 21104, 21113 (SP 274096; SP 274108); munic. de Três Lagoas, reserva Agroflorestal, sobre raízes de árvore, $\pm 230 \mathrm{~m}$ alt., 12/IX/1992, O. Yano \& M.P. Marcelli 17036 (SP 242181); idem, base do tronco de árvore, 12/IX/1992, O. Yano \& M.P. Marcelli 17058 (SP 242203); munic. de Selviria, Faz. do Cacildo, $\pm 40 \mathrm{~km}$ de Ilha Solteira em direção a Três Lagoas, no solo pantanoso entre fanerógamos, perto do cerradão, 24/X/1984, O. Yano \& N. Taroda 9284 (SP 191905); idem, Córrego da Véstia, sobre tronco podre, 5/XI/1985, O. Yano \& M.R.P. Noronha 9579 (SP 206284); Campo Grande, reserva Biológica, Univ. Federal de MS, base do tronco de arbusto, 8/IV/1998, O. Yano \& J. Xavier 25280 (SP 322093).

Cresce associada a Lejeunea phyllobola Nees \& Mont.

*Rhacopilopsis trinitensis (Müll. Hal.) E. Britton \& Dixon ex Dixon

Material examinado: BRASIL. Mato Grosso do Sul: munic. de Bonito, ca. 56 $28^{\prime}$ W, $21^{\circ} 8^{\prime}$ S, ca. 300m alt., MS-178 Bonito-Bodoquena, nascente do Rio Sucuri, sobre árvore, margem do rio, 4/VIII/1994, C.E. Rodrigues Jr. \& M.R. Pietrobom-Silva 798 (SP 322863); munic. de Corumbá, Morro Tromba dos Macacos, sobre pedra escorrendo água da cachoeira, $\pm 250 \mathrm{~m}$ alt., 2/XI/1993, O. Yano et al. 21122 (SP 274116).

*Vesicularia vesicularis (Schwägr.) Broth.

Material examinado: BRASIL. Mato Grosso do Sul: munic. de Aparecida do Taboado, ca. $51^{\circ} 05^{\prime} \mathrm{W}$, $20^{\circ} 06$ 'S, ca. 300m alt., rod. BR-158, Ap. do TaboadoParanaíba, ca. $20 \mathrm{~km}$ de Ap. do Taboado, capões de cerradão, sobre tronco em decomposição, 17/II/1996, M.R. Pietrobom-Silva et al. 2834p.p. (SP 322778); munic. de Paranaíba, ca. 51³2'W, $19^{\circ} 37^{\circ}$ 'S, BR-158 Paranaíba-Cassilândia, ca. 5km do Porto Raimundo, capões de mata seca semi decídua, sobre tronco em decomposição, 17/II/1996, M.R. Pietrobom-Silva et al. 2876p.p. (SP 322781); munic. de Costa Rica, ca. $53^{\circ} 13^{\prime} \mathrm{W}, 18^{\circ} 47^{\prime} \mathrm{S}$, povoado Laje, ca. 550m alt., MS-306 Cantina Costa Rica-Capela, cachoeira do Rio Laje, capões de cerrado, sobre barranco rochoso, 20/II/1996, M.R. Pietrobom-Silva et al. 2976p.p. (SP 322808); 
idem, sobre tronco em decomposição, Ribeirão Sucuri, 20/II/1996, M.R. Pietrobom-Silva et al. 2987 (SP 322821); munic. de Pedro Gomes, ca. 54²31'W, $18^{\circ} 4^{\prime}$ 'S, ca. $220 \mathrm{~m}$ alt., rod. Buriti-Pedro Gomes, solo úmido, 6/X/1993, M.R. Pietrobom-Silva \& C.E. Rodrigues Jr. 1126B (SP 322867); Campo Grande, reserva Biológica, Univ. Federal de MS, sobre tronco de árvore na mata úmida, 8/IV/1998, O. Yano \& J. Xavier 25251 (SP 322064).

Cresce associada a Chryso-hypnum diminutinum (Hampe) W.R. Buck, Cyrto-hypnum involvens (Mitt.) W.R. Buck \& H.A. Crum e Philonotis hastata (Duby) Wijk \& Margad.

*Vesicularia vesicularis var. portoricensis (Brid.) W.R. Buck

Material examinado: BRASIL. Mato Grosso do Sul: munic. de Corumbá, Morro Tromba dos Macacos, pendente em raízes na altura da cachoeira, $\pm 250 \mathrm{~m}$ alt., 2/XI/1993, O. Yano et al. 21123 (SP 274117).

*Vesicularia vesicularis var. rutilans (Brid.) W.R. Buck

Material examinado: BRASIL. Mato Grosso do Sul: munic. de Corumbá, Morro Tromba dos Macacos, sobre pedra úmida no riacho, $\pm 230 \mathrm{~m}$ alt., 2/XI/1993, O. Yano et al. 21095, 21098 (SP 274090; SP 274093).

\section{LEUCOBRYACEAE}

Ochrobryum gardneri (Müll. Hal.) Lindb.

Material examinado: BRASIL. Mato Grosso do Sul: munic. de Rochedo (19 $\left.57^{\circ} \mathrm{S}, 5^{\circ} 07^{\prime} \mathrm{W}\right)$, sobre tronco de Curatella americana, em cerradão, 25/I/1979, D.M. Vital 8436 (SP 147508); munic. de Três Lagoas, reserva Agroflorestal, sobre tronco caído na mata, $\pm 230 \mathrm{~m}$ alt., 12/XI/1992, O. Yano \& M.P. Marcelli 17086 (SP 242229); munic. de Selviria, Faz. do Cacildo, $\pm 40 \mathrm{~km}$ de Ilha Solteira em direção a Três Lagoas, base do tronco no cerradão, 25/X/1984, O. Yano \& N. Taroda 9306 (SP 191926); idem, Estação Experimental da UNESP, sobre cortiça de tronco no cerradão, 7/XI/1985, O. Yano \& M.R. Pereira-Noronha 9721 (SP 206426); idem, sobre tronco no cerradão, 7/XI/1985, O. Yano \& M.R. Pereira-Noronha 9722, 9748 (SP 206427; SP 206452); idem, Faz. Agropecuária Santa Maria, sobre tronco podre no cerradão, 7/XI/1985, O. Yano \& M.R. Pereira-Noronha 9752 (SP 206456).
Octoblepharum albidum Hedw. var. albidum

Material examinado: BRASIL. Mato Grosso do Sul: munic. de Costa Rica, ca. 53 ${ }^{\circ} 13^{\prime} \mathrm{W}, 18^{\circ} 47^{\prime} \mathrm{S}$, povoado Laje, ca. 550m alt., MS-306 Cantina Costa Rica-Capela, cachoeira do Rio Laje, sobre tronco de árvore, 20/II/1996, M.R. Pietrobom-Silva et al. 2978p.p. (SP 322811); munic. de Ribas do Rio Pardo, base de árvore no cerradão, 25/I/1979, O. Yano 1332 (SP 147466); munic. de Aquidauana, encosta da serra Santa Bárbara $\left(20^{\circ} 37^{\prime} \mathrm{S}, 55^{\circ} 37^{\prime} \mathrm{W}\right)$, sobre rochas areníticas, 13/XII/1979, D.M. Vital 8612 (SP 147899); munic. de Corumbá, sobre tronco de bacuri, 2/XII/1979, D.M. Vital 8616 (SP 147903); munic. de Três Lagoas, vegetação de cerrado entre a Usina de Jupiá e a cidade, sobre tronco, 25/VIII/1983, O. Yano \& R.C. Compagnoli 8300 (SP 189736); idem, reserva Agroflorestal, base do tronco de árvore, $\pm 230 \mathrm{~m}$ alt., 12/IX/1992, O. Yano \& M.P. Marcelli 17019 (SP 242164); munic. de Selviria, Faz. Estação Experimental da UNESP, $\pm 18 \mathrm{~km}$ de Ilha Solteira em direção a Ap. do Tabuado, sobre tronco no cerradão, 22/X/1984, O. Yano \& J. Semir 9246 (SP 191867); idem, Faz. do Cacildo, $\pm 40 \mathrm{~km}$ de Ilha Solteira em direção a Três Lagoas, base do tronco no cerradão, 23/X/1984, O. Yano \& N. Taroda 9272, 9304 (SP 191893; SP 191924); idem, Faz. Agropecuária Santa Maria, sobre tronco no cerradão, 7/XI/1985, $O$. Yano \& M.R. Pereira-Noronha 9751 (SP 206455); idem, sobre tronco queimado no cerradão, 7/XI/1985, O. Yano \& M.R. Pereira-Noronha 9754 (SP 206458); Campo Grande, reserva Biológica, Univ. Federal de MS, sobre tronco de arbusto, 8/IV/1998, $O$. Yano \& J. Xavier 25274 (SP 322087); munic. de Corumbá, Faz. Nhorimim, Reserva Salina do Oito, tronco de Acuri, 10/IX/1988, V.I. Pott et al. 567 (SP 280982).

Cresce associada a Lejeunea flava (Sw.) Nees.

* Octoblepharum albidum var. violacens Müll. Hal.

Material examinado: BRASIL. Mato Grosso do Sul: munic. de Costa Rica, ca. 53 $13^{\circ}$ 'W, $18^{\circ} 47^{\prime}$ 'S, povoado Laje, ca. 550m alt., MS-306 Cantina Costa Rica-Capela, cachoeira do Rio Laje, capões de cerrado, entre raízes, 20/II/1996, M.R. Pietrobom-Silva et al. 2975a (SP 322805); munic. de Corumbá, Morro Tromba dos Macacos, base do tronco de Acuri na mata, $\pm 210 \mathrm{~m}$ alt., 2/XI/1993, O. Yano et al. 21090 (SP 274085).

*Octoblepharum cocuiense Mitt.

Material examinado: BRASIL. Mato Grosso do

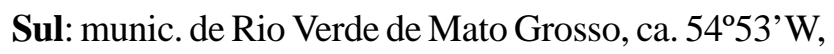


$18^{\circ} 55^{\prime}$ S, ca. $400 \mathrm{~m}$ alt., Serra Pimenteira, cachoeira do Cervo, barranco úmido, 22/II/1994, C.E. Rodrigues Jr. \& M.R. Pietrobom-Silva 736 (SP 322857).

*Octoblepharum cylindricum Schimp. ex Mont.

Material examinado: BRASIL. Mato Grosso do Sul: munic. de Cassilândia, ca. 51 $37^{\circ} \mathrm{W}, 19^{\circ} 07^{\prime} \mathrm{S}$, ca. 400m alt., MS-306 Cassilândia-Chapadão do Sul, ca. $5 \mathrm{~km}$ de Cassilândia, capões de cerrado, sobre tronco em decomposição, 18/II/1996, M.R. Pietrobom-Silva et al. 2898 (SP 322782).

*Octoblepharum pulvinatum (Dozy \& Molk.) Mitt.

Material examinado: BRASIL. Mato Grosso do Sul: munic. de Indaiá do Sul, ca. 52 $23^{\circ} \mathrm{W}, 18^{\circ} 57^{\prime} \mathrm{S}$, ca. $500 \mathrm{~m}$ alt., cachoeira aos fundos da cidade, capões de cerrado, sobre tronco de árvore, 18/II/1996, M.R. Pietrobom-Silva et al. 2945 (SP 322800); munic. de Corumbá, Morro Tromba dos Macacos, paredão úmido da cachoeira, $\pm 250 \mathrm{~m}$ alt., 2/XI/1993, O. Yano et al. 21128 (SP 274122).

\section{LEUCODONTACEAE}

Pseudocryphyaea domingensis (Spreng.) W.R. Buck

Material examinado: BRASIL. Mato Grosso do Sul: munic. de Dourados, Faz. do Sr. Antonio Tonani, sobre casca de árvore na mata, 25/I/1979, A.I. Milanez s.n. (SP 147460).

\section{METEORIACEAE}

*Papillaria deppei (Hornsch.) Müll. Hal.

Material examinado: BRASIL. Mato Grosso do Sul: munic. de Costa Rica, ca. 53 ${ }^{\circ} 13^{\prime} \mathrm{W}, 18^{\circ} 47^{\prime} \mathrm{S}$, ca. $450 \mathrm{~m}$ alt., Ribeirão Sucuruí, aos fundos da cidade de Costa Rica, sobre tronco de árvore, 20/II/1996, M.R. Pietrobom-Silva et al. 2984 (SP 322818).

Papillaria nigrescens (Hedw.) A. Jaeger

Material examinado: BRASIL. Mato Grosso do Sul: munic. de Cassilândia ca. $51^{\circ} 37^{\prime} \mathrm{W}, 1^{\circ} 07^{\prime} \mathrm{S}$, ca. 400m alt., MS-306 Cassilândia-Chapadão do Sul, ca. $5 \mathrm{~km}$ de Casilândia, capões de cerrado, sobre tronco de árvore, 18/II/1996, M.R. Pietrobom-Silva et al. 2901p.p. (SP 322785); munic. de Indaiá do Sul, ca. $52^{\circ} 23^{\prime} \mathrm{W}, 18^{\circ} 57^{\prime} \mathrm{S}$, ca. $500 \mathrm{~m}$ alt., cachoeira aos fundos da cidade, capões de cerrado, sobre tronco de árvore, 18/II/1996, M.R. Pietrobom-Silva et al. 2944p.p., 2948 (SP 327993; SP 322803); munic. de Ribas do Rio Pardo, 71km de Campo Grande/Ribas do
Rio Pardo, sobre pau podre perto do rio, 25/I/1979, O. Yano 1327 (SP 147464); munic. de Jardim, sobre tronco, ca. 52km da cidade, 28/XI/1979, D.M. Vital 8575 (SP 147876); munic. de Três Lagoas, reserva Agroflorestal, ninho de pássaro, $\pm 230 \mathrm{~m}$ alt., 12/IX/1992, O. Yano \& M.P. Marcelli 17102 (SP 242244); Novo Mundo, perto da cachoeira, sobre tronco de Leguminosa, 18/III/1982, O. Yano 4033p.p. (SP 174215).

Cresce associada a Chryso-hypnum elegantulum (Hook.) Hampe, Frullania ericoides (Nees) Mont. e Zelometeorium patulum (Hedw.) Manuel.

Zelometeorium patulum (Hedw.) Manuel

Material examinado: BRASIL. Mato Grosso do Sul: munic. de Cassilândia, ca. 51 ${ }^{\circ} 37^{\prime} \mathrm{W}, 1^{\circ} 07^{\prime} \mathrm{S}$, ca. 400m alt., MS-306 Cassilândia-Chapadão do Sul, ca. $5 \mathrm{~km}$ de Cassilândia, capões de cerrado, sobre tronco de árvore, 18/II/1996, M.R. Pietrobom-Silva et al. 2900, 2901p.p. (SP 322784; SP 322785); munic. de Indaiá do Sul (= Chapéu Azul), ca. $52^{\circ} 23^{\prime} \mathrm{W}, 18^{\circ} 57^{\prime} \mathrm{S}$, ca. $500 \mathrm{~m}$ alt., cachoeira aos fundos da cidade, capões de cerrado, sobre tronco de árvore, 18/II/1996, M.R. Pietrobom-Silva et al. 2934p.p. (SP 322790); munic. de Alto Taquari, ca. 53 ${ }^{\circ} 17^{\prime} \mathrm{W}, 17^{\circ} 50^{\prime} \mathrm{S}$, ca. $600-650 \mathrm{~m}$ alt., ca. $25 \mathrm{~km}$ a sudeste da cidade, Córrego da Laje, paredão rochoso úmido, 21/II/1996, M.R. PietrobomSilva et al. 3013p.p. (SP 322838); munic. de Dourados, Faz. do Sr. Antonio Tonani, sobre casca de árvore na mata, 25/I/1979, A.I. Milanez s.n. (SP 147461).

Cresce associada a Cheilolejeunea trifaria (Reinw. et al.) Mizut., Lophocolea bidentata (L.) Dumort., Neckeropsis undulata (Hedw.) Reichardt e Papillaria nigrescens (Hedw.) A. Jaeger.

*Zelometeorium recurvifolium (Hornsch.) Manuel

Material examinado: BRASIL. Mato Grosso do

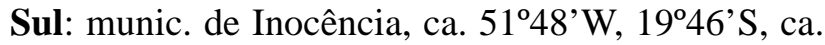
$400 \mathrm{~m}$ alt., rod. MS São Pedro-Inocência, ca. $17 \mathrm{~km}$ de Inocência, cerrado, sobre tronco de árvore, 11/XI/1995, M.R. Pietrobom-Silva \& M. de Lucca Jr. 2477p.p. (SP 322773).

Cresce associada a Neckeropsis undulata (Hedw.) Reichardt.

\section{NECKERACEAE}

Neckeropsis undulata (Hedw.) Reichardt

Material examinado: BRASIL. Mato Grosso do Sul: munic. de Inocência, ca. 51 $48^{\circ} \mathrm{W}, 19^{\circ} 46^{\prime} \mathrm{S}$, ca. $400 \mathrm{~m}$ alt., rod. MS São Pedro-Inocência, ca. $17 \mathrm{~km}$ de Inocência, cerrado, sobre tronco de árvore, 11/XI/1995, 
M.R. Pietrobom-Silva \& M. de Lucca Jr. 2477p.p. (SP 322773); munic. de Indaiá do Sul, ca. 52²3' W, $18^{\circ} 57^{\prime} \mathrm{S}$, ca. $500 \mathrm{~m}$ alt., cachoeira aos fundos da cidade, capões de cerrado, sobre tronco de árvore, 18/II/1996, M.R. Pietrobom-Silva et al. 2937, 2939 (SP 322792; SP 322794); munic. de Costa Rica, ca. 531' 'W, $18^{\circ} 47^{\prime} \mathrm{S}$, ca. $450 \mathrm{~m}$ alt., Ribeirão Sucuri, aos fundos da cidade de Costa Rica, sobre tronco em decomposição, 20/II/1996, M.R. Pietrobom-Silva et al. 2982 (SP 322816); munic. de Bonito, ca. 56 $28^{\circ} \mathrm{W}, 21^{\circ} 08^{\prime} \mathrm{S}$, ca. 500-600m alt., MS-178 Bonito-Bodoquena, Gruta do Lago Azul, ca. $20 \mathrm{~km}$ da cidade, sobre árvore, 4/VIII/1994, C.E. Rodrigues Jr. \& M.R. PietrobomSilva 775 (SP 322860); idem, Ilha do Padre, Rio Formosa, sobre tronco, 4/VIII/1994, C.E. Rodrigues Jr. \& M.R. Pietrobom-Silva 790 (SP 322862); munic. de Alto Taquari, ca. $53^{\circ} 17^{\prime} \mathrm{W}, 17^{\circ} 50^{\prime} \mathrm{S}$, ca. $600-650 \mathrm{~m}$ alt., ca. $25 \mathrm{~km}$ à sudeste da cidade, Córrego da Laje, paredão rochoso úmido, 21/II/1996, M.R. PietrobomSilva et al. 3013p.p., 3016 (SP 322840; SP 322838); Dourados, sobre tronco podre na mata, 25/I/1979, A.I. Milanez s.n. (SP 147462); munic. de Jardim, sobre pau grosso, 28/XI/1979, D.M. Vital 8585 (SP 147882).

Cresce associada a Zelometeorium patulum (Hedw.) Manuel e Z. recurvifolium (Hornsch.) Manuel.

*Pinnatella minuta (Mitt.) Broth.

Material examinado: BRASIL. Mato Grosso do Sul: munic. de Costa Rica, ca. 53 ${ }^{\circ} 13^{\prime} \mathrm{W}, 18^{\circ} 47^{\prime}$ 'S, ca. $450 \mathrm{~m}$ alt., Ribeirão Sucuruí, aos fundos da cidade de Costa Rica, sobre tronco em decomposição, 20/II/1996, M.R. Pietrobom-Silva et al. 1981 (SP 322815).

*Porotrichum longirostre (Hook.) Mitt.

Material examinado: BRASIL. Mato Grosso do Sul: munic. de Alto Taquari, ca. $53^{\circ} 17^{\prime} \mathrm{W}, 17^{\circ} 50^{\prime} \mathrm{S}$, ca. 600-650m alt., ca. 25km à sudeste da cidade, Córrego da Laje, paredão rochoso úmido, 21/II/1996, M.R. Pietrobom-Silva et al. 3012 (SP 322823); idem, ca. $53^{\circ} 17^{\prime} \mathrm{W}, 17^{\circ} 50^{\prime} \mathrm{S}$, ca. $600-650 \mathrm{~m}$ alt., ca. $25 \mathrm{~km}$ a sudeste da cidade, paredão rochoso úmido, 21/II/1996, M.R. Pietrobom-Silva et al. 3011p.p. (SP 322837).

Cresce associada a Bryopteris filicina (Sw.) Nees e Lejeunea autoica R.M. Schust.

\section{ORTHOTRICHACEAE}

*Macromitrium altituberculosum Bartr. ex Grout

Material examinado: BRASIL. Mato Grosso do Sul: munic. de Dourados, $2 \mathrm{~km}$ além do rio Vacaria na estrada Campo Grande-Dourados, sobre pau podre no capão da mata, 25/I/1979, A.I. Milanez s.n. (SP 147457); munic. de Bonito, sobre tronco de Embiraçu, 28/XI/1979, D.M. Vital 8591 (SP 147885); munic. de Selviria, Faz. Agropecuária Santa Maria, sobre tronco no cerradão, 7/XI/1985, O. Yano \& M.R. Pereira-Noronha 9730 (SP 206435).

\section{PLAGIOTHECIACEAE}

*Pilosium chlorophyllum (Hornsch.) Müll. Hal.

Material examinado: BRASIL. Mato Grosso do Sul: munic. de Indaiá do Sul, ca. $52^{\circ} 23^{\prime} \mathrm{W}, 18^{\circ} 57^{\prime} \mathrm{S}$, ca. $500 \mathrm{~m}$ alt., cachoeira aos fundos da cidade, capões de cerrado, sobre tronco de árvore, 18/II/1996, M.R. Pietrobom-Silva et al. 2943 (SP 322798); munic. de Bonito, ca. 56 $28^{\prime} \mathrm{W}, 21^{\circ} 8^{\prime} \mathrm{S}$, ca. 500-600m alt., MS-178 Bonito-Bodoquena, abismo Anhumas, ca. $20 \mathrm{~km}$ da cidade, rupícola, próximo da boca do abismo, 4/VIII/1994, M.R. Pietrobom-Silva \& C.E. Rodrigues Jr. 1326 (SP 322869); munic. de Alto Taquari, ca. $53^{\circ} 17^{\prime} \mathrm{W}, 17^{\circ} 50^{\prime} \mathrm{S}$, ca. $600-650 \mathrm{~m}$ alt., ca. $2 \mathrm{~km}$ a sudeste da cidade, Córrego da Laje, paredão rochoso úmido, 21/II/1996, M.R. Pietrobom-Silva et al. 3014p.p., 3019 (SP 322839; SP 322843).

Cresce associada a Thuidium tomentosum Schimp. in Besch.

\section{POTTIACEAE}

*Barbula indica (Hook.) Spreng.

Material examinado: BRASIL. Mato Grosso do Sul: munic. de Bonito, ca. 56 $28^{\prime} \mathrm{W}, 21^{\circ} 8^{\prime} \mathrm{S}$, ca. $300 \mathrm{~m}$ alt., MS-178 Bonito-Bodoquena, rio Formoso, cachoeira próximo da estrada, barranco úmido, 4/VIII/1994, C.E. Rodrigues Jr. \& M.R. Pietrobom-Silva 786p.p. (SP 322861).

Cresce associada a Marchantia papillata Raddi.

\section{*Hyophila involuta (Hook.) A. Jaeger}

Material examinado: BRASIL. Mato Grosso do Sul: munic. de Ribas do Rio Pardo, 16km antes da cidade, base do tronco, perto do rio, 25/I/1979, O. Yano 1346 (SP 147477); munic. de Aquidauana, sobre rocha arenítica na base da serra Santa Bárbara, 3/XII/1979, D.M. Vital 8608 (SP 147896); munic. de Corumbá, Morro Tromba dos Macacos, sobre pedra, 2/XI/1993, O. Yano et al. 21037 (SP 274035).

*Molendoa sendtneriana (B.S.G.) Limpr.

Material examinado: BRASIL. Mato Grosso do 
Sul: munic. de Rochedo, sobre rochas areníticas, em cerrado, 25/I/1978, D.M. Vital 8427 (SP 147502).

*Pseudosymblepharis schimperiana (Par.) H.A. Crum

Material examinado: BRASIL. Mato Grosso do Sul: munic. de Indaiá do Sul, ca. $52^{\circ} 23^{\prime} \mathrm{W}, 18^{\circ} 57^{\prime} \mathrm{S}$, ca. $500 \mathrm{~m}$ alt., cachoeira aos fundos da cidade, sobre tronco de árvore, capões de cerrado, 18/II/996, M.R. Pietrobom-Silva et al. 2941 (SP 322796).

*Tortella humilis (Hedw.) Jenn.

Material examinado: BRASIL. Mato Grosso do Sul: munic. de Bonito, sobre barranco ao longo do ribeirão Formoso, 28/XI/1979, D.M. Vital 8587 (SP 147883).

*Tuerckheimia guatemalensis Broth.

Material examinado: BRASIL. Mato Grosso do Sul: munic. de Alto Taquari, ca. $53^{\circ} 17^{\prime} \mathrm{W}, 17^{\circ} 50^{\prime} \mathrm{S}$, ca. $600-650 \mathrm{~m}$ alt., ca. $25 \mathrm{~km}$ a sudeste da cidade, barranco úmido, 21/II/1996, M.R. Pietrobom-Silva et al. 3021 (SP 322845).

\section{PTEROBRYACEAE}

Jaegerina scariosa (Lor.) Arz.

Material examinado: BRASIL. Mato Grosso do Sul: munic. de Aparecida do Taboado, ca $51^{\circ} 05^{\prime} \mathrm{W}$, $20^{\circ} 06$ 'S, ca. 300m alt., rod. BR-158 Ap. do TaboadoParanaíba, ca. 20km de Ap. do Taboado, capões de cerradão, sobre tronco de árvore, 17/II/1996, M.R. Pietrobom-Silva et al. 2831 (SP 322775).

*Pireella pohlii (Schwägr.) Card.

Material examinado: BRASIL. Mato Grosso do Sul: munic. de Cassilândia, ca. 51'37'W, $19^{\circ} 07^{\prime}$ 'S, ca. 400m alt., MS-306 Cassilândia-Chapadão do Sul, ca. 5km de Cassilândia, capões de cerrado, sobre tronco de árvore, 18/II/1996, M.R. Pietrobom-Silva et al. 2903 (SP 322786); munic. de Rio Verde de Mato Grosso, ca. $54^{\circ} 53^{\prime} \mathrm{W}, 18^{\circ} 55^{\prime}$ 'S, ca. $400 \mathrm{~m}$ alt., Serra Pimenteira, cachoeira do Cervo, barranco úmido, 22/II/1994, C.E. Rodrigues Jr. \& M.R. Pietrobom-Silva 735 (SP 322856); munic. de Jardim, sobre pau grosso, 28/XI/1979, D.M. Vital 8584 (SP 147881).

\section{RACOPILACEAE}

Racopilum tomentosum (Hedw.) Brid.

Material examinado: BRASIL. Mato Grosso do
Sul: munic. de Costa Rica, ca. $53^{\circ} 13^{\prime} \mathrm{W}, 18^{\circ} 47^{\prime} \mathrm{S}$, ca. $450 \mathrm{~m}$ alt., Ribeirão Sucuruí, aos fundos da cidade de Costa Rica, sobre tronco em decomposição, 20/II/1996, M.R. Pietrobom-Silva et al. 2986p.p. (SP 322820); munic. de Alto Taquari, ca. $53^{\circ} 17^{\prime} \mathrm{W}, 17^{\circ} 50^{\prime} \mathrm{S}$, ca. $600-650 \mathrm{~m}$ alt., ca. $25 \mathrm{~km}$ a sudeste da cidade, córrego da Laje, paredão rochoso úmido, 21/II/1996, M.R. Pietrobom-Silva et al. 3013p.p. (SP 322838); munic. de Corumbá, Morro Tromba dos Macacos, sobre pedra exposta na mata, $\pm 210 \mathrm{~m}$ alt., 2/XI/1993, O. Yano et al. 21092 (SP 274087).

Cresce associada a Neckeropsis undulata (Hedw.) Reichardt e Plagiochila disticha (Lehm. \& Lindenb.) Mont.

\section{RHACHITHECIACEAE}

*Jonesiobryum cerradense Vital ex Allen \& Pursell

Material examinado: BRASIL. Mato Grosso do Sul: munic. de Rochedo, sobre tronco de Qualea sp., em cerradão, 25/I/1979, D.M. Vital 8435, A.P. LuiziPonzo (SP 147507); Campo Grande, sobre tronco de Licania sp., 27/I/1979, D.M. Vital 8443 (SP 147515); munic. de Rio Brilhante, sobre tronco de Licania sp., 27/XI/1979, D.M. Vital 8559 (SP 147863); munic. de Três Lagoas, vegetação cerrado entre a Usina de Jupiá e a cidade, sobre tronco de árvore, 25/VIII/1989, $O$. Yano \& R.C. Compagnoli 8299 (SP 189735); idem, reserva Agroflorestal, sobre tronco de Qualea sp., $\pm 230 \mathrm{~m}$ alt., 12/IX/1992, O. Yano \& M.P. Marcelli 17067 (SP 242212); munic. de Selviria, Faz. Estação Experimental da UNESP, $\pm 18 \mathrm{~km}$ de Ilha Solteira em direção a Ap. do Tabuado, sobre tronco, no cerradão, 22/X/1984, O. Yano \& J. Semir 9249 (SP 191869); idem, entre os ritidomas da árvore do cerradão, 22/X/1984, O. Yano \& J. Semir 9257 (SP 191878); idem, Faz. do Cacildo, $\pm 40 \mathrm{~km}$ de Ilha Solteira em direção a Três Lagoas, na base do tronco no cerradão, 23/X/1984, O. Yano \& N. Taroda 9265 (SP 191886); idem, entre os ritidomas do tronco no cerradão, 23/X/1984, O. Yano \& N. Taroda 9267, 9279, 9296 (SP 191888; SP 191900; SP 191917); idem, Estação Experimental da UNESP, sobre tronco no cerradão, 7/XI/1985, O. Yano \& M.R. Pereira-Noronha 9716 (SP 206421); idem, Faz. Agropecuária Santa Maria, sobre cortiça de tronco no cerradão, 7/XI/1985, $O$. Yano \& M.R. Pereira-Noronha 9732 (SP 206436); idem, sobre tronco queimado no cerradão, 7/XI/1985, O. Yano \& M.R. Pereira-Noronha 9745 (SP 206449). 


\section{SEMATOPHYLLACEAE}

*Sematophyllum caespitosum (Hedw.) Mitt.

Material examinado: BRASIL. Mato Grosso do Sul: munic. de Ribas do Rio Pardo, $16 \mathrm{~km}$ antes da cidade, margem do rio, 25/I/1979, O. Yano 1343p.p., 1344, 1345 (SP 133226; SP 147475; SP 147476); munic. de Dourados, $2 \mathrm{~km}$ além do Rio Vacaria na estrada Campo Grande-Dourados, chão do capão da mata, 25/I/1979, A.I. Milanez s.n. (SP 147454; SP 147455; SP 147456); Campo Grande, sobre tronco de Sapindaceae, 24/I/1979, D.M. Vital 8415 (SP 147496); munic. de Três Lagoas, reserva Agroflorestal, sobre tronco de árvore, $\pm 230 \mathrm{~m}$ alt., 12/IX/1992, O. Yano \& M.P. Marcelli 17039 (SP 242184); idem, sobre galhos de arbusto, $\pm 230 \mathrm{~m}$ alt., 12/IX/1992, O. Yano \& M.P. Marcelli 17047 (SP 242192); idem sobre liana na mata, 12/IX/1992, O. Yano \& M.P. Marcelli 17066 (SP 242211); idem na base do tronco na mata, 12/IX/1992, O. Yano \& M.P. Marcelli 17089 (SP 242231); munic. de Selviria, Estação Experimental da UNESP, sobre cortiça do tronco vivo no cerradão, 7/XI/1985, O. Yano \& M.R. Pereira-Noronha 9719 (SP 206424); Campo Grande, reserva Biológica, Univ. Federal de MS, sobre tronco de árvore na mata úmida, 8/IV/1998, O. Yano \& J. Xavier 25257 (SP 322070).

*Sematophyllum cuspidiferum Mitt.

Material examinado: BRASIL. Mato Grosso do Sul: munic. de Costa Rica, ca. 53 ${ }^{\circ} 13^{\prime} \mathrm{W}, 18^{\circ} 47^{\prime} \mathrm{S}$, povoado Laje, ca. 550m alt., MS-306 Cantina Costa Rica-Capela, cachoeira do Rio Laje, capões de cerrado, sobre paredão rochoso, 20/II/1996, M.R. Pietrobom-Silva et al. 2974 (SP 322804); munic. de Ribas do Rio Pardo, $\pm 71 \mathrm{~km}$ depois do Campo Grande, barranco perto do rio, 25/I/1979, O. Yano 1329 (SP 147465).

*Sematophyllum subsimplex (Hedw.) Mitt.

Material examinado: BRASIL. Mato Grosso do Sul: munic. de Cassilândia, ca. 51 ${ }^{\circ} 37^{\prime} \mathrm{W}, 1^{\circ} 07^{\prime} \mathrm{S}$, ca. 400m alt., MS-306 Cassilândia-Chapadão do Sul, ca. $5 \mathrm{~km}$ de Cassilândia, margem da rodovia, capões de cerrado, sobre tronco em decomposição, 18/II/1996, M.R. Pietrobom-Silva et al. 2899 (SP 322783); munic. de Três Lagoas, reserva Agroflorestal, sobre tronco caído na mata, $\pm 230 \mathrm{~m}$ alt., 12/IX/1992, O. Yano \& M.P. Marcelli 17084 (SP 242227); idem, sobre tronco podre na mata, 12/IX/1992, O. Yano \& M.P. Marcelli 17117 (SP 242256).
*Taxithelium planum (Brid.) Mitt.

Material examinado: BRASIL. Mato Grosso do Sul: munic. de Costa Rica, ca. 53 ${ }^{\circ} 13^{\prime} \mathrm{W}, 18^{\circ} 47^{\prime} \mathrm{S}$, ca. $450 \mathrm{~m}$ alt., Ribeirão Sucuruí, aos fundos da cidade de Costa Rica, sobre bloco rochoso, úmido, 20/II/1996, M.R. Pietrobom-Silva et al. 2985 (SP 322819).

\section{STEREOPHYLLACEAE}

*Entodontopsis leucostega (Brid.) W.R. Buck \& Ireland

Material examinado: BRASIL. Mato Grosso do Sul: munic. de Brasilândia, Lagoa Machado, ca. $52^{\circ} 07^{\prime} \mathrm{W}, 21^{\circ} 43^{\prime} \mathrm{S}, \mathrm{ca} .7 \mathrm{~km}$ da sede pela trilha turística, sobre tronco em decomposição, 24/IX/1996, M.R. Pietrobom-Silva 3528 (SP 322824); munic. de Corumbá, Morro Tromba dos Macacos, sobre tronco de Mangifera, $\pm 210 \mathrm{~m}$ alt., 2/XI/1993, O. Yano et al. 21035 (SP 274033); idem, sobre estipe de Bocaiúva, $\pm 210 \mathrm{~m}$ alt., 2/XI/1993, O. Yano et al. 21083p.p. (SP 274078); munic. de Três Lagoas, rreserva Agroflorestal, sobre liana na mata, $\pm 230 \mathrm{~m}$ alt., 12/IX/1992, O. Yano \& M.P. Marcelli 17028 (SP 242173); munic. de Selviria, Córrego da Véstia, base do tronco, 5/XI/1985, O. Yano \& M.R.P. Noronha 9611 (SP 206316); idem, Estação Experimental da UNESP, base do tronco no cerradão, 7/XI/1985, O. Yano \& M.R. Pereira-Noronha 9720, 9723 (SP 206425; SP 206428); idem, Faz. Agropecuária Santa Maria, sobre tronco no cerradão, 7/XI/1985, O. Yano \& M.R. Pereira-Noronha 9740 (SP 206444); Campo Grande, reserva Biológica, Univ. Federal de MS, sobre tronco de arbusto na mata úmida, 8/IV/1998, O. Yano \& J. Xavier 25250 (SP 322063); idem, base do tronco de arbusto, 8/IV/1998, O. Yano \& J. Xavier 25269 (SP 322082).

Cresce associada a Schiffneriolejeunea polycarpa (Nees) Gradst.

*Entodontopsis nitens (Mitt.) W.R. Buck \& Ireland

Material examinado: BRASIL. Mato Grosso do Sul: munic. de Rochedo (20 $08^{\circ} \mathrm{S}, 5^{\circ} 04^{\prime} \mathrm{W}$ ), barrancos do rio Aquidauana, 25/I/1979, D.M. Vital 8417 (SP 147498); munic. de Corumbá, Morro Tromba dos Macacos, sobre pedra úmida no riacho, $\pm 230 \mathrm{~m}$ alt., 2/XI/1993, O. Yano et al. 21094p.p. (SP 274089).

Stereophyllum radiculosum (Hook.) Mitt.

Material examinado: BRASIL. Mato Grosso do Sul: munic. de Corumbá, morro do Urucum (19²12'S, 
$\left.57^{\circ} 38^{\prime} \mathrm{W}\right)$, sobre tronco caído na margem da estrada para o morro, 29/I/1979, O. Yano 1354 (SP 147484); munic. de Jardim, sobre tronco, em mata de vale, 28/XI/1979, D.M. Vital 8576 (SP 147877); munic. de Corumbá, Morro Tromba dos Macacos, sobre tronco de Mangifera, $\pm 210 \mathrm{~m}$ alt., 2/XI/1993, O. Yano et al. 21033 (SP274031); idem, sobre tronco de arbusto, 2/XI/1993, O. Yano et al. 21112 (SP 274107); munic. de Três Lagoas, reserva Agroflorestal, sobre tronco de árvore na mata, $\pm 230 \mathrm{~m}$ alt., $12 / \mathrm{IX} / 1992$, O. Yano \& M.P. Marcelli 17104 (SP 242246); munic. de Selviria, mata ciliar do Córrego da Véstia, sobre tronco, 5/XI/1985, O. Yano \& M.R.P. Noronha 9575, 9593, 9605 (SP 206280; SP 206298; SP 206310).

\section{THUIDIACEAE}

Cyrto-hypnum involvens (Mitt.) W.R. Buck \& H.A. Crum

Material examinado: BRASIL. Mato Grosso do Sul: munic. de Aparecida do Taboado, ca. $51^{\circ} 05^{\prime} \mathrm{W}$, $20^{\circ} 06$ 'S, ca. 300m alt., rod. BR-158 Ap. do TaboadoParanaíba, ca. $20 \mathrm{~km}$ de Ap. do Taboado, capões de cerradão, sobre tronco em decomposição, 17/II/1996, M.R. Pietrobom-Silva et al. 2834p.p. (SP 322778); munic. de Costa Rica, ca. 5313'W, 18 $47^{\circ}$ 'S, ca. 450m alt., Ribeirão Sucuri, aos fundos da cidade de Costa Rica, sobre tronco em decomposição, 20/II/1996, M.R. Pietrobom-Silva et al. 2988p.p. (SP 322822).

Cresce associada a Chryso-hypnum diminutivum (Hampe) W.R. Buck e Vesicularia vesicularis (Schwägr.) Broth.

*Cyrto-hypnum schistocalyx (Müll. Hal.) W.R. Buck \& H.A. Crum

Material examinado: BRASIL. Mato Grosso do Sul: munic. de Aparecida do Taboado, ca. 51 $05^{\prime} \mathrm{W}$, $20^{\circ} 06$ 'S, ca. $300 \mathrm{~m}$ alt., BR-158 Ap. do TaboadoParanaíba, ca. 20km de Ap. do Taboado, capões de cerradão, sobre tronco de peroba, 17/II/1996, M.R. Pietrobom-Silva et al. 2836 (SP 322780).

*Thuidium tomentosum Schimp. in Besch.

Material examinado: BRASIL. Mato Grosso do Sul: munic. de Indaiá do Sul, ca. $52^{\circ} 23^{\prime} \mathrm{W}, 18^{\circ} 57^{\prime} \mathrm{S}$, ca. $500 \mathrm{~m}$ alt., cachoeira aos fundos da cidade, capões de cerrado, sobre tronco em decomposição, 18/II/1996, M.R. Pietrobom-Silva et al. 2936, 2938 (SP 322791; SP 322793); munic. de Costa Rica, ca. 53²13'W, $18^{\circ} 47^{\prime} \mathrm{S}$, povoado de Laje, ca. 550m alt., MS-306 Cantina Costa Rica-Capela, cachoeira do Rio Laje, capões de cerrado, sobre barranco rochoso, 20/II/1996, M.R. Pietrobom-Silva et al. 2976a (SP 322806); munic. de Alto Taquari, ca. $53^{\circ} 17^{\prime} \mathrm{W}, 17^{\circ} 50^{\prime} \mathrm{S}$, ca. $600-650 \mathrm{~m}$ alt., ca. $25 \mathrm{~km}$ a sudeste da cidade, estrada de acesso à Fazenda Córrego da Laje, paredão rochoso úmido, 21/II/1996, M.R. Pietrobom-Silva et al. 3014p.p. (SP 322839).

Cresce associada a Pilosium chlorophyllum (Hornsch.) Müll. Hal.

Os Estados da região Centro-Oeste brasileiro são pouco estudados em relação às briófitas. Para o Estado de Mato Grosso do Sul havia referências de apenas 61 espécies, sendo uma de antócero, 18 de hepáticas e 42 de musgos; para o Mato Grosso foram citadas 179 espécies, sendo 37 de hepáticas e 142 de musgos; para Goiás 204, sendo um de antócero, 41 de hepáticas e 162 de musgos; para o Distrito Federal, 79 espécies sendo 13 de hepáticas e 66 de musgos e para o Tocantins, que anteriormente pertencia à região CentroOeste, têm-se referidas apenas 32 espécies, sendo cinco de hepáticas e 27 espécies de musgos.

O levantamento feito, ou melhor, o estudo das coleções realizados por várias pessoas aumentou em muito o número de espécies de briófitas para o Estado de Mato Grosso do Sul passando a 161 espécies, sendo dois de antóceros, 58 de hepáticas e 101 de musgos, aumentando $162,3 \%$ o número de espécies, ampliando ainda o conhecimento sobre a distribuição das espécies já mencionadas para o Estado. Portanto, fazendo-se coletas intensivas nas regiões de serra e nas matas de galeria, com certeza o número da diversidade de briófitas deverá aumentar.

\section{Agradecimentos}

Os autores agradecem à curadora Dra. Neusa Taroda Ranga, do Herbarium Riopretense (SJRP), da UNESP, Campus de São José do Rio Preto, pela doação das exsicatas de material briofítico ao Herbário do Estado Maria Eneyda P. Kauffmann Fidalgo, São Paulo (SP).

\section{Referências}

Bartram, E.B. 1949. Mosses of Guatemala. Fieldiana Botany 25: $1-442$.

Bischler, H. 1969. Le genre Leptolejeunea (Spruce) Steph. en Amerique. Nova Hedwigia 17: 265-350.

Bischler, H.; Bonner, C.E.B. \& Müller, H.A. 1963. Studies in Lejeuneaceae VI: the genus Microlejeunea Steph. in Central and South America. Nova Hedwigia 5(1-2): 359-411. 
Buck, W.R. 1983. A synopsis of the South American taxa of Fabronia (Fabroniaceae). Brittonia 35(3): 248-254.

Florschütz, P.A. 1964. The mosses of Suriname. part 1. Leiden, E.J. Brill. 1-271.

Frahm, J.-P. 1991. Dicranaceae: Campylopodioideae, Paraleucobryoideae. Flora Neotropica, monograph 54: 1-238.

Fulford, M.H. 1976. Manual of the leafy Hepaticae of Latin America-part IV. Memoirs of the New York Botanical Garden 11(4): 393-535.

Görts-Van Rijn, A.R.A. (ed.). 1996. Flora of the Guiana. Musci III. Ser. C: Bryophytes 1: 363-490.

Gradstein, R.S. 1994. Lejeuneaceae: Ptychantheae, Brachiolejeuneae. Flora Neotropica, monograph 62: $1-216$.

Hässel de Menéndez, G.G. 1962. Estudio de las Anthocerotales y Marchantiales de la Argentina. Opera Lilloana 7: 1-297.

Hasegawa, J. 1994. New classification of Anthocerotae. The Journal of the Hattori Botanical Laboratory 76: 21-34.

Hell, K.G. 1969. Briófitas talosas dos arredores da cidade de São Paulo (Brasil). Boletim da Universidade de São Paulo, Botânica 25: 1-187.

Ireland, R.R. \& Buck, W.R. 1994. Stereophyllaceae. Flora Neotropica, monograph 65: 1-49.

Lemos-Michel, E. \& Yano, O. 1998. O gênero Bryopteris (Hepatophyta) no Brasil. Acta Botanica Brasilica 12(1): 5-24.

Lindenberg, J.B.G. 1844. Monographia Hepaticarum generis Plagiochilae. Bonnae, Sumptibus Henry \& Cohen.

Manuel, M.G. 1977. A monograph of the genus Zelometeorium Manuel, gen. nov. (Bryopsida: Meteoriaceae). The Journal Hattori Botanical Laboratory 43: 107-126.

Ochi, H. 1980. A revision of the Neotropical Bryoideae, Musci (First Part). The Journal of the Faculty of Education Tottori University, nat. science 29(2): 49-154.

Reese, W.D. 1993. Calymperaceae. Flora Neotropica, monograph 58: 1-102.

Reiner-Drehwald, M.E. 2000. Las Lejeuneaceae (Hepaticae) des Misiones, Argentina VI. Lejeunea y Taxilejeunea. Tropical Bryology 19: 81-131.
Schuster, R.M. 1980. The Hepaticae and Anthocerotae of North America east of the hundredth meridian. Columbia University Press, New York.

Schuster, R.M. 1984. Evolution, phylogeny and classification of the Hepaticae. In: R.M. Schuster (ed.). New Manual of Bryology 2: 892-1070.

Scremin-Dias, E.; Pott, V.J.; Hora, R.C. \& Souza, P.R. (orgs.). 1999. Nos jardins submersos da Bodoquena: guia para identificação de plantas aquáticas de Bonito e região. Ed. UFMS, Campo Grande.

Sharp, A.J., Crum, H. \& Heckel, P. 1994. The moss flora of Mexico. Memoirs of the New York Botanical Garden 69: $1-1113$.

Spence, J.R. 1996. Rosulabryum genus novum (Bryaceae). The Bryologist 99(2): 221-225.

Stotler, R.E. \& Crandall-Stotler, B. 1974. A monograph of the genus Bryopteris (Swartz) Nees von Esenbeck. Bryophytorum Bibliotheca 3: 1-159.

Vanden Berghen, C. 1976. Frullaniaceae (Hepaticae) africanae. Bulletin du Jardin Botanique National de Belgique 46: 1-220.

Vitt, D.H. 1984. Classification of the Bryopsida. In: R.M. Schuster (ed.). New Manual of Bryology 2: 696-759.

Yano, O. 1979. Contribuição ao inventário dos Musci brasileiros: Helicophyllaceae. Rickia 8: 7-16.

Yano, O. 1981. A checklist of Brazilian mosses. The Journal of the Hattori Botanical Laboratory 50: 279-456.

Yano, O. 1984a. Checklist of Brazilian liverworts and hornworts. The Journal of the Hattori Botanical Laboratory 56: 481-548.

Yano, O. 1984b. Contribuição ao inventário dos Musci brasileiros: 3. Racopilaceae (Bryopsida, Isobryales). Revista Brasileira Botânica 7(1): 57-63.

Yano, O. 1989. An additional checklist of Brazilian bryophytes. The Journal of the Hattori Botanical Laboratory 66: 371-434.

Yano, O. 1992. Leucobryaceae (Bryopsida) do Brasil. Tese de Doutorado. Universidade de São Paulo.

Yano, O. 1995. A new additional annotated checklist of Brazilian bryophytes. The Journal of the Hattori Botanical Laboratory 78: 137-182.

Zander, R.H. 1993. Genera of the Pottiaceae: mosses of harsh environments. Buletin of the Buffalo Society of Natural Sciences 32: 1-378. 